\title{
Gauge Theory in Witten's Approach to the Generation Problem
}

\author{
Brett McInnes \\ Department of Mathematics, National University of Singapore, 10 Kent Ridge Crescent 0511, \\ Republic of Singapore
}

Received August 6, 1990

\begin{abstract}
In Witten's topological theory of the generation problem, gauge groups are identified with the $E_{8}$ centraliser of the holonomy group of the internal manifold. Here we show that this amounts to interpreting gauge groups as generalised symmetry groups of the (internal) Levi-Civitá connection. We then give techniques for computing centralisers in exceptional groups, taking into account the fact that holonomy groups are frequently disconnected. These techniques allow us to deal with compact locally irreducible Ricci-flat Riemannian manifolds of all holonomy types and dimensions.
\end{abstract}

\section{Introduction}

In view of the recent experimental work on determining the number of light generations of fermions, it is timely to reconsider how the matter lies from a theoretical standpoint. How are we to understand the existence of the two apparently superfluous generations? Candelas et al. (1985) proposed that the answer lies in the deep structure of the Dirac equation, in the relationship between its solutions and the topology of (multi-dimensional) space-time. This profound and beautiful approach to the generation problem leads to the simple relation

$$
\#=\frac{1}{2}|\chi+\tau|,
$$

where \# is the number of generations, and $\chi$ and $\tau$ are respectively the Euler characteristic and the signature of the internal manifold (Green et al., 1987). These ideas are almost invariably associated with 10-dimensional string theory; but this is not really necessary, as the account given by Green et al. (1987) makes quite clear. Indeed, Witten (1985) sketched the main ideas well before the advent of the heterotic string. In view of the importance of the problem, it seems to us that the approach of Witten (1985) and Candelas et al. (1985) is worthy of study in its own right, independent of its association with string theory. In this work, then, we shall study the foundations of this approach, regarding it mainly as a contribution 
to the theory of the generation problem - though the methods and results are also relevant to string theory. For this reason, we do not confine attention to Kähler manifolds or to six internal dimensions.

The central technical device leading to the above formula for \# is usually described by the unfortunate title of "embedding the (linear) connection in the gauge group." The main reason for the general tendency to associate this idea with string theory is the fact that it is a natural way of satisfying the anomaly cancellation condition. But it is also - independently - a natural way of obtaining a non-trivial gauge field on the internal manifold, which is necessary to solve the chirality problem in higher dimensional theories. "Embedding the connection in the gauge group" is therefore of considerable interest beyond the string theoretic applications.

It is clear from these remarks (for the details, see Green et al., 1987) that this approach to the generation problem depends strongly on topological aspects of gauge theory. At the same time, however, we shall argue that "embedding the connection" throws a new light on the meaning of gauge symmetry itself. Of course, one of the main virtues of the original multi-dimensional theories of the Kaluza-Klein type was precisely their ability to elucidate the real meaning of gauge "symmetries." What is a gauge "symmetry?" Kaluza and Klein reply: a gauge group, $J$, is nothing but a group of symmetries - isometries - of the internal manifold. Although the Kaluza-Klein theories have their drawbacks, the idea of finding a concrete interpretation of gauge groups as some kind of geometric symmetry obviously has a strong appeal.

Within the original framework given by Candelas et al. (1985), gauge groups arise in ways which appear to have little in common with the Kaluza-Klein interpretation. The manifolds employed in superstring compactifications are Ricci-flat, compact, and have non-zero Euler characteristics. They have therefore no non-trivial Killing vector fields, and so there can of course be no question of interpreting the gauge group as a group of isometries. In fact, as we saw earlier, the chirality problem is solved by assuming that there are non-zero gauge fields on the internal manifold itself. To be more precise: one begins with the assumption that all fields are assigned to an irreducible representation of the group $E_{8}$. (Actually, $E_{8} \times E_{8}$, but we can ignore the second $E_{8}$ henceforth.) Then the linear holonomy group of the internal manifold is interpreted as the holonomy group of an $E_{8}$ gauge field, and the "observable" gauge group arises as the subgroup of $E_{8}$ that consists of elements commuting with every element of the holonomy group. In the case of the holonomy group $S U(3)$, this subgroup (the centraliser of $S U(3)$ ) is isomorphic to $E_{6}$. Notice that $E_{8}$ and $E_{6}$ play different roles - the latter is to be the "observed" gauge group, but it is $E_{8}$ that unifies the fields in a single representation. The two are linked by the holonomy group of the internal manifold, $M$ : in this way, the geometry of $M$ dictates the gauge interactions of the theory, just as the topology of $M$ determines the generation structure.

The formalism presents us with the following interpretation of the gauge group: it is the $G$-centralizer of the (internal) manifold holonomy group, where $G$ is the group that unifies all interactions. But this raises two further problems. Firstly, this is a merely formal interpretation of the gauge group, $J$ - we have lost the more meaningful interpretation of $J$ as an automorphism group of a geometric structure. Secondly, we now have a technical problem: how does one go about computing the centralisers of subgroups of $G$ ? The objective of this work is to propose techniques for dealing with these questions. 
We shall argue that the "centraliser" interpretation of $J$ is in fact extremely closely allied to the "isometry" interpretation. The idea of embedding the holonomy group of $M$ (which we denote by $\Psi(M)$ ) in some larger group $G$ is evidently a simple generalization of the fact that $\Psi(M)$ is naturally a subgroup of the orthogonal group $O(n), n=\operatorname{dim} M$. Obviously, this calls for an equally simple generalization of Riemannian geometry. When this is duly constructed, it quickly becomes clear that both interpretations of $J$ amount to regarding it as an automorphism group of the linear connection of $M$. In short, the "stringmotivated" account of gauge theory given above is quite as geometric as the Kaluza-Klein approach.

As for the second problem: granted that $J$ is the $G$-centraliser of $\Psi(M)$, we clearly wish to know which subgroups of $G$ can occur as the centraliser of some $\Psi(M)$. In general, this is no trivial exercise. Firstly, we must consider the fact that many subgroups of $G$ cannot be expressed as the centraliser of any other subgroup of $G$ - given $J \subset G$, there may exist no subgroup $A \subset G$ such that $C A=J$, where $C A$ henceforth denotes the centraliser of $A$ in $G$. Secondly, even if $A$ does exist, there may exist no Riemannian manifold $M$ with holonomy group isomorphic to $A$, particularly if - as would normally be the case in a physical application - $M$ is subject to some geometric constraint such as Ricci flatness. Finally, and most importantly for the present work, the concrete problem of actually computing $C \Psi(M)$, given $G$ and $\Psi(M)$ explicitly, can be surprisingly subtle. This is most emphatically the case when $G$ is an exceptional group. Notice that we are speaking here of computing the full subgroup of those elements of the group $E_{8}$ that centralise the holonomy group of $\mathrm{M}$ : the problem is intrinsically group - theoretic, and is not always reducible to Lie-algebra computations. In general, the centraliser $C A$ is sensitive to the global structure of $A$-connected subgroups frequently have different centralisers to disconnected ones, and so on. Again, the topology of $\Psi(M)$ is of basic importance in holonomy theory; for example, a Ricci-flat fourdimensional Riemannian manifold can have $S U(2)$ as its holonomy group, but never $S O(3)$. These facts are commonly disregarded, with the result that some statements on these matters are distinguished rather by their optimism than by the confidence they inspire.

For example, the assertion that the centraliser of $S U(3)$ in $E_{8}$ is isomorphic to $E_{6}$ is generally taken to be obviously valid. The embedding is allegedly through the " $S U(3) \times E_{6}$ " subgroup of $E_{8}$. If this were indeed the correct global form of the subgroup in question, then we could conclude, by inspection alone, only the following: that $E_{8}$ contains an $E_{6}$ which centralises $S U(3)$. We could not deduce that $E_{6}$ was the full centraliser of $S U(3)$ - indeed, that would evidently not be the case, since the centraliser would contain $\mathbb{Z}_{3} \times E_{6}$, where $\mathbb{Z}_{3}$ is the centre of $S U(3)$. Under these assumptions, then, $C(S U(3))$ has at least one discrete factor, but it could have many more - one simply cannot settle this by inspection. Any temptation to declare that disconnected groups are of no interest should be firmly resisted, as the theory of cosmic strings (for example) clearly shows (Vilenkin, 1985).

The reader will perhaps be relieved to learn that the centraliser of $S U(3)$ in $E_{8}$, is, in fact, precisely $E_{6}$; there are no finite factors. The necessity of proving this, however, should now be clear. There are less innocuous examples: the assertion (Green et al., 1987) that superstring compactification on manifolds of SO(6) holonomy leads to an $S O(10)$ grand unification group is, quite simply, false. Unambiguous techniques for computing centralisers are obviously needed here. 
Completely systematic methods for computing $C \Psi(M)$ for arbitrary $G$ and $M$ do not seem to exist. We shall mainly concentrate on the case $G=E_{8}$, partly because of its topical interest in connection with superstrings, partly because it is the most interesting of the exceptional groups, and partly because the techniques we shall use to deal with $E_{8}$ can readily be adapted to handle less complicated groups.. Similarly, we shall require $M$ to be compact and Ricci-flat (and to satisfy a few other minor technical constraints). The extension to the case of non-Ricci-flat manifolds is interesting but very lengthy, and involves few conceptual novelties.

We begin, however, by substantiating the claim that the "centraliser interpretation" of gauge groups is geometrically natural.

\section{Gauge Groups as Automorphism Groups}

(General references for this section are Kobayashi-Nomizu (1963) and particularly Fischer (1987).)

Let $M$ be a compact connected Riemannian manifold of dimension $n$, and let $O(M)$ be the bundle of orthonormal frames over $M$; its structural group is the orthogonal group $O(n)$. If $\Psi(M)$ is the linear holonomy group of $M$, then the holonomy reduction theorem implies that $O(M)$ admits a sub-bundle $H(M)$ with structural group $\Psi(M)$, and that the linear connection reduces to a connection on $H(M)$. Thus $H(M)$, the holonomy bundle, contains all the information needed to reconstruct the linear connection on $O(M)$. Indeed, it is quite possible to take the position that $H(M)$ is the fundamental object; then the linear connection is regarded as a one-form $\omega$ on $H(M)$ which takes its values in the algebra of $\Psi(M)$. Noticing that $\Psi(M)$ always has a canonical embedding in $O(n)$, one would study the principal $O(n)$-bundles having $H(M)$ as a sub-bundle, using perhaps the fact that $\omega$ induces a connection on every such $O(n)$-bundle. From this point of view, $O(M)$ is but one among a family of $O(n)$-bundles to which we have been led by the fact that $\Psi(M)$ can be embedded in $O(n)$ in a natural way.

Regarding Riemannian geometry in this only slightly unorthodox fashion, we are led to the obvious generalisation: replace $O(n)$ by some other compact Lie group $G$ in which $\Psi(M)$ can be embedded, preferably in some natural manner. Then let $P$ be a principal $G$-bundle over $M$ such that $H(M)$ is a sub-bundle of $P$. Such a bundle always exists (Husemoller, 1975), and the linear connection on $H(M)$ induces a connection on $P$, just as it does on $O(M)$. Under these circumstances, we shall say that the linear holonomy bundle has been extended to a $G$-bundle. This construction permits us to interpret $\omega$ either as a linear connection (when $\Psi(M)$ is regarded as a subgroup of $O(n)$ ) or as a gauge field (when $\Psi(M)$ is regarded as a subgroup of $G$ ). This dual view of $\omega$ is precisely what is meant when one speaks, in string theory (Green et al., 1987), of "embedding the (linear) connection in the gauge group." More generally - that is, beyond the applications to string theory it is certainly interesting to construct higher-dimensional theories with the principal purpose of finding a topological explanation of the generation structure. Such theories require (Witten, 1985) topologically non-trivial gauge fields on the internal manifold, and again the most natural way to obtain these is to "embed the connection in the gauge group." The above construction is relevant to all such theories.

To recapitulate: we assume as usual that space-time has the structure \{Observed 4-manifold $\times M$, where $M$ is a compact, connected Riemannian 
manifold. The holonomy bundle $H(M)$ is extended to a $G$-bundle, $(P, M, G)$, where $G$ is the group that unifies all interactions, and where the embedding of the holonomy group, $\Psi(M) \rightarrow G$, is specified. The linear connection on $M$ extends to a connection on $P$; this gives a rigorous formulation of "embedding the linear connection in the gauge group" and so paves the way to a solution of the chirality problem in higher-dimensional theories.

The idea of studying principal bundles that admit frame bundles as sub-bundles is actually extremely natural from a purely mathematical point of view. To see this, let $P$ be an arbitrary $G$-bundle over $M$, and let $\operatorname{Aut}(P)$ be the group of all automorphisms of $P$. There is a natural homomorphism $\alpha$ from $\operatorname{Aut}(P)$ onto $\operatorname{Diff}(M)$, the diffeomorphism group of $M$, defined by

$$
\alpha(\mu)(x)=\pi(\mu(p)),
$$

where $x \in M, \mu \in \operatorname{Aut}(P), \pi$ is the projection map and $p$ is any element of $P$ such that $\pi(p)=x$. The kernel of $\alpha$ is clearly

$$
\operatorname{VAut}(P)=\{\mu \in \operatorname{Aut} P \text { such that } \pi \circ \mu=\pi\},
$$

the so-called group of vertical automorphisms of $P$. Therefore $\operatorname{VAut}(P)$ is a normal subgroup of $\operatorname{Aut}(P)$, and furthermore $\operatorname{Aut}(P) / \operatorname{VAut}(P)=\operatorname{Diff}(M)$.

In group-theoretic language, this means that $\operatorname{Aut}(P)$ is an extension of $\operatorname{Diff}(M)$. The first question to ask under these circumstances is this: does the extension split? (The extension is said to split if $\operatorname{Aut}(P)$ has a subgroup 4 , isomorphic to $\operatorname{Diff}(M)$, such that

and

$$
\operatorname{Aut}(P)=\Delta \cdot \operatorname{VAut}(P)
$$

$$
\Delta \cap \operatorname{VAut}(P)=\{\text { identity automorphism }\} \text {. }
$$

Here and henceforth, $K \cdot L$ means (if $K, L$ are subgroups of some group) the set $\{k l$, where $k \in K, l \in L\}$.) In general, of course, the answer to this question is "no"-we cannot expect to "solve" the above relation so easily. The extension splits if and only if there is a global homomorphic cross-section $\sigma: \operatorname{Diff}(M) \rightarrow \operatorname{Aut}(P)$, with $\alpha \circ \sigma$ $=$ identity, and of course $\sigma$ will not usually exist. However, there is one particularly natural case in which it does, as we shall now explain.

Suppose that $P$ admits the full linear frame bundle $F(M)$ as a subbundle. $(F(M)$ is the $G L(n, \mathbb{R})$-bundle of all frames over $M$, the appropriate object here since we do not yet wish to discuss connections.) Then a homomorphic cross-section $\sigma: \operatorname{Diff}(M) \rightarrow \operatorname{Aut}(P)$ is easily constructed. Let $f \in \operatorname{Diff}(M)$ and let $u \in F(M)$. We regard $u$ as a non-singular linear map from $\mathbb{R}^{n}$ to the tangent space at $\pi(u)$. We define $\sigma(f)$ to be the natural lift of $f$ to $F(M)$; that is, $\sigma(f)$ is the $F(M)$ automorphism defined by

$$
\sigma(f)(u) \xi=f_{*}(u \xi),
$$

where $\xi$ is any element of $\mathbb{R}^{n}$ and $f_{*}$ denotes the differential of $f$, so that $u \xi$ is a tangent vector at $\pi(u)$ and $f_{*}(u \xi)$ is a tangent vector at $f(\pi(u))$; thus $\sigma(f)(u)$ is a frame at $f(\pi(u)$ ). Then $\sigma(f)$ is indeed an automorphism of $F(M)$, and hence of $P$, because any element of $P$ can be expressed as $u g$ for some $u \in F(M)$ and $g \in G$, so we can define $\sigma(f)(u g)=[\sigma(f)(u)] g$. The fact that $\sigma$ is a homomorphism is just the "chain rule":

$$
\begin{aligned}
\sigma\left(f_{1} \circ f_{2}\right)(u) \xi & =f_{1 *}\left(f_{2 *}(u \xi)\right)=f_{1 *}\left(\sigma\left(f_{2}\right)(u) \xi\right) \\
& =\sigma\left(f_{1}\right)\left(\sigma\left(f_{2}\right)(u)\right) \xi
\end{aligned}
$$


that is $\sigma\left(f_{1} \circ f_{2}\right)=\sigma\left(f_{1}\right) \circ \sigma\left(f_{2}\right)$. Finally, if $x \in M$ and $u \in F(M)$ with $\pi(u)=x$, then we know that $\sigma(f)(u)$ is a frame at $f(\pi(u))=f(x)$ for any $f \in \operatorname{Diff}(M)$, so that

$$
\alpha(\sigma(f))(x)=\pi(\sigma(f)(u))=f(x),
$$

whence $\alpha(\sigma(f))=f$ and so $\alpha \circ \sigma=$ identity. Hence the extension splits in this case and we can write

$$
\operatorname{Aut}(P)=[\sigma(\operatorname{Diff}(M))] \cdot \operatorname{VAut}(P) .
$$

We see, then, that the idea of $P$ admitting a frame bundle as a sub-bundle arises quite naturally in the study of $\operatorname{Aut}(P)$.

Of course, $\operatorname{Aut}(P)$ will not usually split as an extension of $\operatorname{Diff}(M)$ - requiring $P$ to admit $F(M)$ as a sub-bundle is a very strong condition. We are interested in the far less restrictive case in which $H(M)$, rather than $F(M)$, is a sub-bundle of $P$. Now whereas every diffeomorphism of $M$ lifts to an automorphism of $F(M)$, not every such natural lift will preserve the structure of $H(M)$; and similarly for VAut $(P)$. (To be precise: an automorphism of $F(M)$ or of $P$ may be said to "preserve the structure" of $H(M)$ if it maps the latter to another holonomy bundle - that is, if every element of $\mu[H(M)]$ can be connected to every other element by a horizontal curve.) Since $H(M)$ is defined by the Levi-Civita connection $\omega$, the obvious way to ensure that $\mu$ preserves the structure of $H(M)$ is to impose the condition $\mu^{*} \omega=\omega$; we leave it to the reader to verify that this has the desired effect. The analogue of $\sigma(\operatorname{Diff}(M))$ is therefore the group

$$
\begin{aligned}
E(\omega)=\{ & \mu \in \operatorname{Aut}(F(M)) \text { such that } \mu \text { is the natural lift of a } \\
& \text { diffeomorphism of } \left.M, \text { and } \mu^{*} \omega=\omega\right\},
\end{aligned}
$$

while the analogue of $\operatorname{VAut}(P)$ is of course

$$
I(\omega)=\left\{\mu \in \operatorname{VAut}(P) \text { such that } \mu^{*} \omega=\omega\right\},
$$

where we are using $\omega$ to denote either the linear connection on $H(M)$ or the induced connections on $F(M)$ and $P$. Clearly $E(\omega)$ and $I(\omega)$ may both be regarded as symmetry groups of $\omega$. We shall call them the exterior and interior symmetry groups, respectively, because every element of $I(\omega)$ induces the identity diffeomorphism on $M$, while no non-trivial element of $E(\omega)$ does so. From a general point of view, however, these distinctions are not very important; $E(\omega)$ is the symmetry group of $\omega$ when the latter is regarded as a linear connection, while $I(\omega)$ is the symmetry group of $\omega$ when we think of it as a $G$ gauge field.

The relevance of all this to our present concerns will be revealed by the following result.

Theorem 2.1. Let $M$ be a compact, connected Riemannian manifold which is not locally isometric to a product of lower-dimensional manifolds, and let $\operatorname{dim}(M)>1$. Let $\omega$ be the Levi-Civitá connection on $M$, and suppose that the holonomy bundle $H(M)$ is a sub-bundle of a specified G-bundle. Then the exterior and interior symmetry groups of $\omega$ can be characterised as follows:

(a) $E(\omega)$ is isomorphic to the isometry group of $M$.

(b) $I(\omega)$ is isomorphic to the $G$-centraliser of the holonomy group of $M$.

Proof. (a) Kobayashi and Nomizu (1963) show that the group of natural lifts of diffeomorphisms of $M$ satisfying $\mu^{*} \omega=\omega$ is isomorphic to the group of affine 
symmetries of $M$. These are diffeomorphisms $f: M \rightarrow M$ which map every parallel vector field along an arbitrary curve $\gamma$ to a parallel field along $f \circ \gamma ;$ in other words, they are symmetries of the connection rather than of the metric. Evidently, then, $E(\omega)$ contains a subgroup isomorphic to the isometry group, but it is not clear (and not true in general) that every element of $E(\omega)$ is an isometry. That is the case, however, if (Kobayashi and Nomizu (1963), p. 242) (i) $\operatorname{dim}(M)>1$, and (ii) $M$ is complete, and (iii) the holonomy group acts irreducibly on the tangent spaces of $M$. In the present case, (i) is valid by assumption, (ii) follows from compactness, and (iii) follows from the assumption that $M$ is not locally isometric to a product, together with the local version of the de Rham splitting theorem (see Besse, 1987). Hence $E(\omega)$ is isomorphic to the isometry group of $M$ under these circumstances.

(b) When $\omega$ is regarded as a connection on $H(M)$, its holonomy group is of course $\Psi(M)$, the holonomy group of $M$. The induced connection on $P$ therefore also has a holonomy group isomorphic to $\Psi(M)$. Now quite generally, for any connection on an arbitrary principal bundle $P$, the subgroup of $\operatorname{VAut}(P)$ satisfying $\mu^{*} \omega=\omega$ is isomorphic (Fischer, 1987) to the $G$-centraliser of the holonomy group. Hence, in our case, $I(\omega)$ is isomorphic to $C \Psi(M)$. This completes the proof.

Notice that it follows immediately from this theorem that both $I(\omega)$ and $E(\omega)$ are finite-dimensional.

The close similarity of the Kaluza-Klein and the "string-inspired" interpretations of the "observed" gauge group $J$ is now obvious. Both kinds of theory interpret $J$ as a group of symmetries of the linear connection of the internal manifold $M$. In Kaluza-Klein theories, $P$ is taken to be the orthonormal frame bundle $O(M)$, and attention is focussed on the isometry group $E(\omega)$; the interior symmetry group is ignored. In string compactifications, $M$ has only a finite group of isometries and so $E(\omega)$ is ignored; but as we have seen, the extension of $H(M)$ to an $E_{8}$ bundlenecessitated by the "embedding of the linear connection in the gauge group" automatically gives rise to a new symmetry group $I(\omega)=C \Psi(M)$. This leads automatically to gauge fields on $M$ (and hence ultimately on four-dimensional space-time). To see this, recall that the canonical metric on $P$ is

$$
\pi^{*} g+k(\omega, \omega),
$$

where $g$ is the metric on $M$ and $k$ is the Cartan-Killing form on $G$ (which we take to be compact and semi-simple). Now every $\mu \in I(\omega)$ satisfies $\pi \circ \mu=\pi$ and $\mu^{*} \omega=\omega$, so

$$
\begin{aligned}
\mu^{*}\left[\pi^{*} g+k(\omega, \omega)\right] & =(\pi \circ \mu)^{*} g+k\left(\mu^{*} \omega, \mu^{*} \omega\right) \\
& =\pi^{*} g+k(\omega, \omega) .
\end{aligned}
$$

Thus $I(\omega)$ acts isometrically on $P$ and hence manifests itself as a gauge group on $M$.

Let us summarise. We have argued that the interpretation of $J$ as the centraliser of the holonomy group actually amounts to a geometric interpretation in the spirit of the Kaluza-Klein approach. The principal distinction lies in the kind of bundle automorphism on which emphasis is placed, but the symmetrical object is the same in each case: namely, the linear connection of the internal manifold, $M$. The real differences lie elsewhere, in the solution of the chirality problem.

Having shown that the interpretation of the observed gauge group as $C \Psi(M)$ is both natural and geometrically meaningful, we may turn to practical matters: how is $C \Psi(M)$ to be computed, especially if $G$ is an exceptional group? What are the possibilities for $J$ ? 


\section{Basic Methods of Centraliser Theory}

Let $G$ be a compact connected Lie group, and let $H$ be a compact but possibly disconnected subgroup. We wish to determine the full centraliser of $H$ in $G$. When $G$ and $H$ can be explicitly represented as groups of matrices, this is often possible by inspection; otherwise, however, there can be a number of difficulties. For example, even if both $G$ and $H$ are connected, one cannot deduce that $C H$ is connected. To see this, take $G=S O(5), H=S O(3)$ embedded in the obvious way. Then $C H$ is not $S O(2)$, but rather the group of matrices

$$
\left[\begin{array}{cc} 
\pm I_{3} & 0 \\
0 & m_{2}
\end{array}\right]
$$

where $m_{2} \in O(2)$, and the \pm sign is chosen according to the determinant of $m_{2}$. Clearly $\mathrm{CH}$ is the disconnected group $O(2)$, despite the fact that both $S O(5)$ and $S O(3)$ are connected. Again, let us consider the centraliser of $O(3)$ in $S O(5)$. We can embed it as

$$
\left[\begin{array}{rrr}
m_{3} & 0 & 0 \\
0 & \pm 1 & 1 \\
0 & 0 & 1
\end{array}\right]
$$

But the centraliser is no longer $O(2)$ or even $S O(2)$ - it is the finite group $\mathbb{Z}_{2} \times \mathbb{Z}_{2}$, generated by $\left[\begin{array}{rrr}-I_{3} & 0 & 0 \\ 0 & +1 & 0 \\ 0 & 0 & -1\end{array}\right]$ and $\left[\begin{array}{ccc}-I_{3} & 0 & 0 \\ 0 & -1 & 0 \\ 0 & 0 & +1\end{array}\right]$. Thus $C(S O(3))$ and $C(O(3))$ are totally different, even though $S O(3)$ is the identity component of $O(3)$. Notice that this example shows that centralisers cannot be reliably computed using Lie algebra techniques alone, since obviously $S O(3)$ and $O(3)$ correspond to the same subalgebra of the algebra of $S O(5)$.

We wish to compute centralisers in $E_{8}$, where such pitfalls cannot be detected by inspection. Therefore we need techniques for proving that centralisers are connected, for dealing with disconnected $H$ when computing $C H$, for understanding questions about the rank of $\mathrm{CH}$, and so on. Such techniques are best explained in the context of particular examples, but there are some general results which can usefully be collected here.

First, the following quite trivial lemma occurs so frequently that we make note of it.

Lemma (3.1). Let $A$ and $B$ be subgroups of any group $G$, such that $A \subset B$. Then $C B \subseteq C A$.

Notice the important distinction between proper inclusion $(A \subset B)$ and inclusion with the possibility of equality.

Second, although it will not be an important issue in our computations, the reader should bear in mind that many subgroups of $G$ cannot be expressed as the centraliser of any other subgroup of $G$. This imposes a fundamental restriction on the range of possibilities for the gauge group. One has, for example, elementary restrictions such as the following.

Lemma (3.2). Let $H \subset G$, and let $Z H, Z G$ denote the respective centres. If $Z H$ does not contain $Z G$, then neither $H$ nor any subgroup of $H$ can be expressed as the $G$-centraliser of some other subgroup of $G$. 
The proof follows easily from Lemma (3.1). Thus, for example, if we embed $S U(m)$ in $S U(n), m<n$, then neither $S U(m)$ nor any of its subgroups can be expressed as a centraliser, because $\mathbb{Z}_{n}$ is not a subgroup of $\mathbb{Z}_{m}$. Hence if we pick $G=S U(n)$ in the discussion of the previous section, then it is impossible to obtain $S U(m)$ as the grand unification group. This is a simple consequence of elementary group theory - it has nothing to do with the geometry of $M$.

Next we state a basic result in Lie theory, which is useful to us because it gives some control over the topology of centralisers.

Theorem (3.3). Let $G$ be a compact, connected Lie group, let $T$ be any torus in $G$, and let $g$ be any element of $C T$. Then there exists a torus in $G$ containing $T$ and $g$.

The proof may be found, for example, in Curtis (1984). This theorem has the following very useful consequence. Let $M_{T}$ denote a maximal torus in $G$ containing $T$, and let $\cup M_{T}$ denote the union of all such. Then the above theorem implies that $C T$ is contained in $\cup M_{T}$. But the mere fact that $M_{T}$ is abelian implies that $\bigcup M_{T}$ is contained in $C T$; hence $C T=\bigcup M_{T}$. But each $M_{T}$ is connected, and they all intersect in $T$. Hence we arrive at the following conclusion.

Theorem (3.4) Let $G$ be a compact, connected Lie group. Then the centraliser of any torus is connected.

For example, the centraliser of $S O(2)$, embedded in any way in any $S O(n)$, is connected; this distinguishes $S O(2)$ sharply from $S O(3)$.

We are now in a position to prove a result which is of crucial importance in dealing with centralisers in the exceptional Lie groups. Although the theorem itself is somewhat technical, the basic strategy is rather simple. Suppose that $H$ is a proper subgroup of a group $G$, and that $C H$ cannot be computed directly - as is usually the case if $G$ is an exceptional Lie group. Now suppose also that we can find a group $K$, with $H \subseteq K \subset G$, such that $C H \subseteq K$. Then clearly $C H=C_{K}(H)$, where $C H$ denotes the centraliser in $G$, while $C_{K}(H)$ denotes the centraliser in $K$. The point, of course, is that it will frequently be very much easier to find $C_{K}(H)$ than $C H$ itself. The following theorem gives simple criteria for the existence of such $K$.

Theorem (3.5). Let $G$ be a compact, connected Lie group and let $H$ be a connected proper subgroup of $G$. Let $K$ be a connected subgroup of $G$ satisfying the following conditions:

(i) $H \subseteq K \subset G$

(ii) $Z \bar{K} \subseteq Z H$

(iii) For any connected subgroup $L$ with $K \subset L \subseteq G, Z K$ is not contained in $Z L$. Then

$$
\mathrm{CH}=\mathrm{C}_{\mathrm{K}}(\mathrm{H}) \text {. }
$$

Proof. First we shall demonstrate that any connected subgroup of $G$ which satisfies condition (iii) can be expressed as

$$
K=C_{0} Z K,
$$

where $C_{0}$ denotes the identity component of the centraliser. To see this, note that obviously $K \subseteq C Z K$, and since $K$ is connected, $K \subseteq C_{0} Z K$. Hence $Z K \subseteq C_{0} Z K$. Obviously every element of $C_{0} Z K$ commutes with every element of $Z K$, so in fact $Z K \subseteq Z C_{0} Z K$. Set $L=C_{0} Z K$. Then we have $K \subseteq L, Z K \subseteq Z L$, and $L$ is connected. By hypothesis, $L$ cannot contain $K$ properly, so we have $K=L=C_{0} Z K$ as asserted. 
Now let $T$ be any maximal torus in $H$. Since $H$ is connected and compact, we can apply Theorem (3.3). From the fact that $T$ is maximal, it follows easily that $Z H \subseteq T$. By hypothesis, we now have $Z K \subseteq T$. Hence we can write

$$
Z K \subseteq T \subseteq H .
$$

Lemma (3.1) now yields

$$
C H \subseteq C T \subseteq C Z K .
$$

But $T$ is also a torus in $G$, and so by Theorem (3.4) $C T$ is connected. (This is the whole purpose of introducing $T$ : it is a subgroup with a centraliser which is known to be connected.) Hence $C T$ is contained not only in $C Z K$ but also in $C_{0} Z K$. Thus $C H \subseteq C_{0} Z K$. But we saw earlier that $C_{0} Z K=K$. Hence every element of $G$ which centralises $H$ is contained in $K$; that is, $C H \subseteq C_{K}(H)$. But obviously $C_{K}(H) \subseteq C H$, and so $C_{K}(H)=C H$. This completes the proof.

We shall now explain some applications of these ideas.

\section{Application: The Centraliser of $S U(3)$ in $E_{8}$}

The grand unification group in string compactifications is identified with the interior symmetry group of the Levi-Civitá connection of a compact manifold with holonomy group $S U(3)$. The holonomy bundle is extended to an $E_{8}$ bundle, where $E_{8}$ is the compact exceptional Lie group of rank 8. We therefore need to know the full centraliser of $S U(3)$ in $E_{8}$. First, however, we need to understand the embedding of $S U(3)$ in $E_{8}$. There are actually several ways of embedding $S U(3)$ in $E_{8}$, and the centraliser depends on the choice. One might argue, for example, that $S U$ (3) only occurs in holonomy theory through its real representation, so that the natural way to embed $S U(3)$ is through $S O(6)$. From a purely group-theoretic point of view, however, there is a more natural embedding which we now describe (and to which we adhere henceforth, because it is the one used by Candelas et al. (1985)).

The algebra $L E_{8}$ (notation: $L G$ denotes the Lie algebra of a Lie group $G$ ) has a maximal sub-algebra isomorphic to $L(S U(3)) \oplus L E_{6}$. This sub-algebra does not generate a subgroup of $E_{8}$ isomorphic to $S U(3) \times E_{6}$, however. The group in question is actually $\left[S U(3) \times E_{6}\right] / \mathbb{Z}_{3}$, where the $\mathbb{Z}_{3}$ is diagonal between the centres of $S U(3)$ and $E_{6}$. The easiest way to see this is to examine the decomposition of the fundamental 248-dimensional representation of $E_{8}$ with respect to this subgroup: in the notation of Slansky (1981), we have

$$
248=(8,1)+(1,78)+(3,27)+(\overline{3}, \overline{2} \overline{7}) .
$$

Now in the 27-dimensional representation of $E_{6}$, the centre must appear (by irreducibility and Schur's lemma) as $\mathbb{Z}_{3}$ multiples of the identity matrix, just as happens in $S U(3)$. The fact that the decomposition contains the term $(3,27)$ therefore implies that the $\mathbb{Z}_{3}$ in $E_{6}$ must be identified, in $E_{8}$, with the $\mathbb{Z}_{3}$ in $S U(3)$. Hence the subgroup in question is indeed $\left[S U(3) \times E_{6}\right] / \mathbb{Z}_{3}$ rather than $S U(3) \times E_{6}$. (This is entirely analogous to the fact that the combined gauge group of electromagnetism and chromodynamics is $[U(1) \times S U(3)] / \mathbb{Z}_{3}$, not $U(1) \times S U(3)$.) Similarly, $E_{8}$ contains $[S U(5) \times S U(5)] / \mathbb{Z}_{5}$ rather than $S U(5) \times S U(5)$; less obviously, $E_{8}$ contains not $S U(9)$ but rather $S U(9) / \mathbb{Z}_{3}$.

In any case, we now have a natural embedding $S U(3) \rightarrow\left[S U(3) \times E_{6}\right] / \mathbb{Z}_{3} \rightarrow E_{8}$. We now wish to compute the full centraliser of $S U(3)$ in $E_{8}$. As pointed out earlier, 
it is rather clear that $C(S U(3))$ contains $E_{6}$, but it is not at all clear that $C(S U(3))$ contains no other element of $E_{8}$. We can settle this with the aid of Theorem (3.5). We take $K=\left[S U(3) \times E_{6}\right] / \mathbb{Z}_{3}$. Then condition (i) of Theorem (3.5) is satisfied. Condition (ii) is satisfied, because $Z(S U(3))=\mathbb{Z}_{3}=\mathrm{ZK}$ in this case. For condition (iii), note that since the algebra of $K$ is maximal in that of $E_{8}$, it follows that $E_{8}$ itself is the only connected subgroup of $E_{8}$ containing $K$ properly. (Note the word "connected"; without this condition, we would need to investigate discrete factors, and the statement would in fact be false. Happily, Theorem (3.5) only requires that we check the connected groups between $K$ and $G$.) Now the centre of $E_{8}$ is $\mathbb{Z}_{1}$, the group consisting of a single element (Helgason, 1978), and this obviously does not contain $\mathbb{Z}_{3}$. Theorem (3.5) now allows us to compute the centraliser in $K$ instead of $E_{8}$; in other words, nothing outside $K$ centralises $S U(3)$. In $K$, the centraliser is obviously $\left[\mathbb{Z}_{3} \times E_{6}\right] / \mathbb{Z}_{3}=E_{6}$. Thus, the centraliser of $S U(3)$ in $E_{8}$ is precisely $E_{6}$. Similarly, $S U(2)$ (embedded through $\left[S U(2) \times E_{7}\right] / \mathbb{Z}_{2}$ ) has centraliser $E_{7}, S U(5)$ has centraliser $S U(5)$, and so on; in each case, one can use Theorem (3.5) to shift the computation from $E_{8}$ to a more tractable subgroup.

It is curious that the centres of $S U(3)$ and $E_{6}$ are not merely isomorphic, but rather identical as subsets of $E_{8}$. As is well known, $E_{6}$ can be broken to (an approximation of) the standard group, $S$, by the "Hosotani mechanism" (Green et al., 1987). This is simply a second application of the above formalism, where now $G$ is taken to be $E_{6}$, and $\omega$ is a flat gauge connection with discrete holonomy group; $S$ is the centraliser of the latter in $E_{6}$. According to Lemma (3.2), therefore, $Z S$ must contain $\mathbb{Z}_{3}$, the centre of $E_{6}$. But the $\mathbb{Z}_{3}$ in the centre of the "standard" group is related to the electric charge assignments of quarks (Chan and Tsou, 1981). On the other hand, the $\mathbb{Z}_{3}$ in the holonomy group is related to the fact that the manifold is 3 complex-dimensional, or has six real dimensions. In a sense, therefore, it can be said that in string compactifications, quark charge assignments are related to the hypothesis that the universe is 10 -dimensional. We leave it to the reader to judge whether this is a satisfactory outcome.

\section{Application: Ranks of Centralisers}

The "standard" gauge group, which governs the electroweak and strong interactions, is of rank 4. Grand unified theories, however, typically involve groups of larger rank, such as $E_{6}$. If we wish to obtain the standard group (with, perhaps, one additional $U(1)$ factor) as a centraliser in $E_{6}$, then it is of interest to study the circumstances under which $\mathrm{CH}$ is not of maximal rank in $G$. For example, Ellis et al. (1988) have studied Calabi-Yau spaces with non-abelian fundamental groups, the objective being to break $E_{6}$ to a subgroup of rank 5. (See also McInnes (1990a).)

Ideally, one should have general results which, given $G$ and $H$, would permit a direct specification of rk $\mathrm{CH}$, the rank of $\mathrm{CH}$. In fact, very little is known in this direction, apart from elementary inequalities such as

$$
\text { rk } C H \leqq \text { rk } G-\text { rk } H+\text { rk } Z H .
$$

In addition, there is apparently a widespread belief that $\mathrm{CH}$ is of maximal rank in $G$ (that is, rk $C H=\mathrm{rk} G$ ) if and only if $H$ is abelian. This is actually incorrect. Let us investigate this question using the methods of Sect. 3. 
We claim that the centraliser of a finite Abelian group in a compact, connected Lie group $G$ can sometimes be of less than maximal rank; so that Hosotani symmetry breaking on a manifold with an abelian fundamental group can break $G$ to a subgroup of lesser rank. The basic result is as follows:

Theorem (5.1). Let $G$ be a compact, connected Lie group, and let $H$ be a subgroup of $G$.

(i) If $\mathrm{H}$ is non-abelian, then $\mathrm{CH}$ cannot be of maximal rank.

(ii) If $\mathrm{H}$ is abelian, and if either $\mathrm{H}$ or $\mathrm{CH}$ is connected, then $\mathrm{CH}$ is of maximal rank.

Proof. (i) Let $H$ be non-abelian and suppose that $C H$ is of maximal rank in $G$. Let $T$ be a maximal torus in $C H$. Then $T \subseteq C H$ and so by Lemma (3.1),

$$
C C H \subseteq C T \text {. }
$$

But $T$ is also a maximal torus in $G$. It follows easily from Theorem (3.3) that $C T=T$. On the other hand, obviously $H \cong C C H$, so we have $H \leqq T$. But $T$ is abelian, so we have a contradiction. Hence $\mathrm{CH}$ cannot be of maximal rank.

(ii) Suppose that $H$ is abelian and that $C H$ is connected. Then $C H$ is closed in $G$, hence compact, and so we can apply Theorem (3.3) to $\mathrm{CH}$. Let $T$ be a maximal torus in $\mathrm{CH}$. Then $T$ contains the centre of $C H, Z C H \cong T$. Since $H$ is abelian, $H \subseteq C H$; in fact, $H \subseteq Z C H$, so $H \subseteq T$. Let $M_{T}$ be a maximal torus in $G$ containing $T$. Then $H \subseteq M_{T}$, so that $C M_{T} \subseteq C H$. But as we saw earlier, $C M_{T}=M_{T}$, so that $M_{T} \subseteq C H$ and $C H$ is of maximal rank. Finally, if $H$ is assumed abelian and connected, then either $\mathrm{CH}=\mathrm{G}$, or $\mathrm{H}$ is a torus so that (Theorem 3.4) $\mathrm{CH}$ is connected. In either case $\mathrm{CH}$ is of maximal rank. This completes the proof.

The only case not covered by this theorem is that in which neither $H$ nor $C H$ is connected, and $H$ is abelian. It is in this case that one can find examples of abelian groups with centralisers of non-maximal rank. In the case of finite abelian groups, we have the following general result. (Recall that the rank of a finite abelian group is the smallest possible number of factors in its expression as a product of cyclic groups.)

Theorem (5.2). Let $G$ be a compact, connected Lie group. Then $G$ admits a finite abelian subgroup $A$ of rank 2 with $C A$ not of maximal rank if and only if there exists $\mathrm{g} \in \mathrm{G}$ such that $\mathrm{Cg}$ is disconnected.

Proof. We use the following lemma.

Lemma (5.3). Let $G$ be a compact, connected Lie group, and let $g \in G$ be such that $C g$ is disconnected. Then there exists $\bar{g} \in G$ such that $C \bar{g}$ is disconnected and $\bar{g}$ is of finite order.

Proof of Lemma. It can be shown (Fischer, 1987) that $C g$ is closed, so it is compact. Therefore $C g$ has a finite number of connected components; thus, $C g / C_{0} g$ is a finite group. Let $m$ be the order of this finite group. Then it is clear that for any $x \in C g$, $x^{m} \in C_{0} g$, so we have a local diffeomorphism $f: C g \rightarrow C_{0} g$ defined by $f: x \rightarrow x^{m}$. Now elements of finite order are dense in $C_{0} g$, because every compact, connected Lie group is covered by its maximal tori (Curtis, 1984). Since $f(x)$ is of finite order if and only if $x$ is of finite order, we can conclude that if $D$ is a connected component of $C g$ other than $C_{0} g$, then $D$ contains an element $\bar{g}$ of finite order. We claim that $C \bar{g}$ is disconnected. For suppose the contrary. Notice first that for any $p \in G, C_{0}(p)$ 
is precisely the union, $\cup M_{p}$, of all maximal tori $M_{p}$ containing $p$; this is a straightforward consequence of Theorem (3.3). Therefore, if $C \bar{g}$ is connected, then $C \bar{g}=\bigcup M_{\bar{g}}$. By definition, $\bar{g} \in C g$, hence $g \in C \bar{g}$, so that $g \in M_{\bar{g}}$ for some maximal torus containing $\bar{g}$. But this means $\bar{g} \in M_{g}$, a maximal torus containing $g$, and so $\bar{g} \in C_{0} g$. But $\bar{g} \in D$, so we have a contradiction. Hence $C \bar{g}$ is disconnected.

Returning to the proof of Theorem (5.2): suppose that $G$ contains an element $g$ such that $\mathrm{Cg}$ is disconnected. By the above lemma, we can assume that $\mathrm{g}$ is of finite order. Let $D$ be a connected component of $C g$ other than $C_{0} g$, and let $h$ be an element of finite order in $D$. Then we claim that the finite abelian (because $h \in D(C g$ ) group $A$ generated by $g$ and $h$ has a centraliser of non-maximal rank. For suppose that $C A$ is of maximal rank. Then a maximal torus $T$ in $C A$ is also maximal in $G$, so $T \cong C A$ implies

$$
A \cong C C A \subseteq C T=T .
$$

Hence $h$ is an element of a maximal torus containing $g$, so $h \in \bigcup M_{g}=C_{0} g$, a contradiction. Hence $C A$ is not of maximal rank. Now obviously the rank of $A$ is either 2 or 1 . But if the rank were 1 , then since $G$ is covered by its maximal tori, there would be a maximal torus containing $A$, and $C A$ would be of maximal rank. The rank of $A$ must therefore be 2 .

Conversely, if $A$ is a finite abelian rank-2 subgroup of $G$, and if $C A$ is not of maximal rank, then $G$ must contain an element $g$ such that $C g$ is disconnected. For let $g, h$ be the generators of $A$, and assume that $C g$ is connected. Then since $h \in C g$, there is a maximal torus containing $g$ and $h$, hence containing $A$; this leads to a contradiction, as above. This completes the proof.

An easy way to find examples of abelian groups with centralisers not of maximal rank is to examine disconnected subgroups $H$ of compact connected Lie groups, such that $H$ can be expressed as $C Z H$. For then $G$ evidently contains $g$ such that $C g$ is disconnected (pick any element of $Z H$ which is not an element of $Z G$ ), and then the above theorem applies. For example, the $O(2)$ subgroup of $S O(3)$ is clearly the centraliser of its centre, and so $S O(3)$ must contain a finite abelian subgroup with centraliser of non-maximal rank. Indeed,

$$
\left\{\left[\begin{array}{lll}
1 & 0 & 0 \\
0 & 1 & 0 \\
0 & 0 & 1
\end{array}\right],\left[\begin{array}{rrr}
-1 & 0 & 0 \\
0 & -1 & 0 \\
0 & 0 & 1
\end{array}\right],\left[\begin{array}{rrr}
1 & 0 & 0 \\
0 & -1 & 0 \\
0 & 0 & -1
\end{array}\right],\left[\begin{array}{rrr}
-1 & 0 & 0 \\
0 & 1 & 0 \\
0 & 0 & -1
\end{array}\right]\right\}
$$

is such a subgroup.

\section{Holonomy Classification Theorems}

We now return to the principal theme of this work. In Sect. 4 we justified the familiar claim that "embedding the connection (of a manifold with holonomy $S U(3)$ ) in the gauge group ( $E_{8}$ )" leads to $E_{6}$ grand unification. Here, however, we regard this as a particular example of a new approach to the generation problem and to the foundations of gauge theory. This example therefore prompts the following question: which subgroups of $E_{8}$ can arise as gauge groups in this picture? Obviously, not every subgroup can be so obtained. As we have seen, there is a purely group-theoretic obstruction - not every subgroup $J$ can be expressed in the form $C A$ for some $A \subset E_{8}$. However, this is a relatively weak obstruction. Far 
stronger is the geometric condition that $A$ should be the holonomy group of some manifold. This is the question to which we now turn: which groups occur as holonomy groups?

For the compact, Ricci-flat manifolds in which we are interested here, no completely satisfactory answer to this question is known (Salamon, 1989). The best that can be done at present is to display lists of "candidate" groups, and to search for examples of manifolds with such groups as holonomy groups. The classical result in this direction is Berger's Theorem (1955). In the case of interest to us, this may be stated as follows. (See also Besse (1987).)

Theorem (6.1). Let $M$ be a connected, simply connected Riemannian manifold with $\operatorname{dim} M=n>1$. Suppose that $M$ is Ricci-flat and is not isometric to a product of lowerdimensional manifolds. Then the holonomy group $\Psi(M)$ must be isomorphic to one of the following:

(i) $S O(n) \quad(n \geqq 4)$,

(ii) $S U(m) \quad(n=2 m, m \geqq 3)$,

(iii) $\operatorname{Sp}(k) \quad(n=4 k, k \geqq 1)$,

(iv) $G_{2} \quad(n=7)$,

(v) $\operatorname{Spin}(7) \quad(n=8)$.

Here $S p(k)$ is the compact symplectic group of $\operatorname{rank} k, G_{2}$ is the exceptional group of rank 2, and Spin(7) is the subgroup of $S O(8)$ isomorphic to the universal covering group of $S O(7)$. For our purposes, the restriction to simply connected manifolds is much too severe. Methods for dealing with the holonomy theory of compact, Ricci-flat, but not simply connected manifolds have been explained elsewhere (McInnes, 1990b). The classification theorem in this case may be stated as follows.

Theorem (6.2). Let $M$ be a compact, connected, Ricci-flat Riemannian manifold with $\operatorname{dim}(M)=n>1$. Suppose that $M$ is not locally isometric to a product of lowerdimensional manifolds and that $M$ is not simply connected. Then $\Psi(M)$ is isomorphic to one of the following groups:

(i) $n \geqq 4$ : (a) $S O(n)$, (b) $O(n)$.

(ii) $n=2 m$, modd, $m \geqq 3$ : (a) $S U(m)$, (b) $S U(m) \rtimes \mathbb{Z}_{2} . n=2 m$, meven, $m \geqq 4$ : (a) $\mathbb{Z}_{2 m} \cdot S U(m)$, (b) $S U(m) \rtimes \mathbb{Z}_{2}$, (c) $\left[\mathbb{Z}_{2 m} \cdot S U(m)\right] \rtimes \mathbb{Z}_{2}$.

(iii) $n=4 k$, k even, $r$ divides $k+1, r \neq 1$ : (a) $\mathbb{Z}_{r} \times S p(k) . n=4 k, k$ odd, $r$ divides $k+1$, $r \neq 1$ : (a) $\mathbb{Z}_{r} \times S p(k)\left(r\right.$ odd), (b) $\mathbb{Z}_{2 r} \cdot S p(k)(r$ even $),(c) Q_{4 r} \cdot S p(k)$, (d) $B_{4 r} \cdot S p(k)(r=6$, 12, 30).

(iv) $n=7:$ (a) $G_{2}$, (b) $\mathbb{Z}_{2} \times G_{2}$.

(v) $n=8$ : (a) $\operatorname{Spin}(7)$.

The proof (McInnes, 1990b) is based on the Cheeger-Gromoll and RiemannRoch theorems. The notation requires some explanation. The symbol $\rtimes$ denotes the semi-direct product; in detail, if $\zeta$ is the generator of $\mathbb{Z}_{2}$, then $\operatorname{Ad}(\zeta)$ acts on $U(m)$ and its subgroups $S U(m)$ and $\mathbb{Z}_{2 m} \cdot S U(m)$ by complex conjugation. For example, in case (ii) (b), $S U(3) \rtimes \mathbb{Z}_{2}$ is a group with two connected components, one of which is $S U(3)$, the other being the set $\{\zeta s, s \in S U(3)\}$, where $\zeta s=\bar{s} \zeta$ for every $s$. The meaning of the dot notation, as in $\mathbb{Z}_{2 m} \cdot S U(m)$, was explained in Sect. 2; but in Theorem (6.2) it can also be interpreted as the direct product factored by the intersection. For example, $\mathbb{Z}_{2 m} \cdot S U(m)$ is isomorphic to $\left[\mathbb{Z}_{2 m} \times S U(m)\right] / \mathbb{Z}_{m}$, where the $\mathbb{Z}_{m}$ is diagonal between $\mathbb{Z}_{2 m}$ and $S U(m)$. This makes it clear that $\mathbb{Z}_{2 m} \cdot S U(m)$ has two connected 
components. Finally, $Q_{4 r}$ and $B_{4 r}$ denote respectively the quaternionic and binary polyhedral groups. These are non-abelian finite groups of order $4 r$, where $Q_{4 r}$ is defined for every integer $r \geqq 2$, but $B_{4 r}$ exists only for $r=6,12,30$. (See, for example, Wolf (1967).) Notice that $Q_{4 r} \cdot S p(k)=\left[Q_{4 r} \times S p(k)\right] / \mathbb{Z}_{2}$, and similarly for $B_{4 r} \cdot S p(k)$. Notice too that $Q_{4 r} \cdot S p(k)$ is not a subgroup of $U(2 k)$, so that a manifold with this holonomy group is not a Kähler manifold, despite the fact that the identity component is $S p(k)$ - which is a subgroup of $U(2 k)$, through $S U(2 k)$. Similar comments hold for manifolds of holonomy $S U(m) \rtimes \mathbb{Z}_{2}$ : these are subgroups of $O(2 m)$ (as for any Riemannian manifold), but not of $U(m)$. On the other hand, $\mathbb{Z}_{2 m} \cdot S U(m), \mathbb{Z}_{r} \times S p(k)$, and $\mathbb{Z}_{2 r} \cdot S p(k)$ are all subgroups of $U(m)$ or $U(2 k)$, so these do correspond to Kähler manifolds.

The groups listed in Theorems (6.1) and (6.2) are the ones of interest to us. Our task is to embed these groups in $E_{8}$ and to compute their centralisers. Again, it must be emphasised that this is a group-theoretic, and not a Lie-algebraic problem. The algebra of $E_{8}$ contains the algebra of $S U(9)$, but it does not follow that the group $E_{8}$ contains the group $S U(9)$ - and, in fact, it does not. The subalgebra must exponentiate to a unique connected subgroup (Helgason, 1978), but this subgroup is $S U(9) / \mathbb{Z}_{3}$. According to Theorems (6.1) and (6.2), none of the manifolds in which we are interested has holonomy $S U(9) / \mathbb{Z}_{3}$; in fact, to belabour the point a little, one can prove that there exists no manifold of any kind with this holonomy group. To take a quite different kind of example: we know that $E_{8}$ does contain $S U(3)$, but it is very far from obvious that it contains $S U(3) \rtimes \mathbb{Z}_{2}$. We shall prove that it does, and we shall see that the centraliser of $S U(3) \rtimes \mathbb{Z}_{2}$ is not $E_{6}$. All of these points must be taken into account. It is advisable to begin with a brief discussion of selected subgroups of the compact exceptional groups.

\section{Some Connected Subgroups of the Exceptional Groups}

A readily accessible source of information on the subalgebras of the exceptional algebras is Slansky (1981). From there we abstract the following (incomplete) list of inclusions. (Recall that $L G$ denotes the Lie algebra of the group $G$.)

$$
\begin{gathered}
L(S U(2)) \oplus L\left(E_{7}\right) \subset L E_{8}, \quad L\left(E_{6}\right) \oplus \mathbb{R} \subset L E_{7}, \\
L(S U(3)) \oplus L\left(E_{6}\right) \subset L E_{8}, \quad L(S U(3)) \oplus L(S U(6)) \subset L E_{7}, \\
L(S U(5)) \oplus L(S U(5)) \subset L E_{8}, \quad L(S p(3)) \oplus L G_{2} \subset L E_{7}, \\
L(S U(9)) \subset L E_{8}, \quad L(S O(10)) \oplus \mathbb{R} \subset L E_{6}, \\
L(S O(16)) \subset L E_{8}, \quad L(S p(4)) \subset L E_{6}, \\
L\left(G_{2}\right) \oplus L\left(F_{4}\right) \subset L E_{8}, \quad L\left(F_{4}\right) \subset L E_{6}, \\
L(S U(2)) \oplus L(S p(3)) \subset L F_{4}, \quad L(S O(4)) \subset L G_{2}, \\
L(S U(2)) \oplus L\left(G_{2}\right) \subset L F_{4}, \quad L(S U(3)) \subset L G_{2}, \\
L(S O(9)) \subset L F_{4} .
\end{gathered}
$$

Each of these inclusions is maximal, in the usual sense. Each subalgebra corresponds to a unique connected subgroup of a given group with an exceptional algebra. The precise structure of these subgroups cannot be deduced from the 
above table alone; one needs also certain representation - theoretic techniques of the kind explained in Sect. 4. The results are as follows:

$$
\begin{gathered}
{\left[S U(2) \times E_{7}\right] / \mathbb{Z}_{2} \subset E_{8}, \quad\left[U(1) \times E_{6}\right] / \mathbb{Z}_{3} \subset E_{7},} \\
{\left[S U(3) \times E_{6}\right] / \mathbb{Z}_{3} \subset E_{8}, \quad[S U(3) \times S U(6)] / \mathbb{Z}_{3} \subset E_{7},} \\
{[S U(5) \times S U(5)] / \mathbb{Z}_{5} \subset E_{8}, \quad S p(3) \times G_{2} \subset E_{7},} \\
S U(9) / \mathbb{Z}_{3} \subset E_{8}, \quad[U(1) \times \operatorname{Spin}(10)] / \mathbb{Z}_{4} \subset E_{6}, \\
\operatorname{Spin}(16) / \mathbb{Z}_{2} \subset E_{8}, \quad S p(4) / \mathbb{Z}_{2} \subset E_{6}, \\
G_{2} \times F_{4} \subset E_{8}, \quad F_{4} \subset E_{6}, \\
{[S U(2) \times S p(3)] / \mathbb{Z}_{2} \subset F_{4}, \quad S O(4) \subset G_{2}, \quad S O(3) \times G_{2} \subset F_{4},} \\
S U(3) \subset G_{2}, \quad \operatorname{Spin}(9) \subset F_{4} .
\end{gathered}
$$

These can either be found in or deduced from the book of Wolf (1967).

One of these inclusions is of such importance - and has been the source of so much confusion - that it requires further discussion. We refer to $\operatorname{Spin}(16) / \mathbb{Z}_{2} \subset E_{8}$. This group is not isomorphic to $S O(16)$. A brief explanation of this fact will allow us to fix our notation for Clifford algebras.

For our purposes, the very simple formulation given by Curtis (1984) is quite adequate. We take the Clifford algebra $C_{k}$ to be generated by elements 1 , $e_{1}, e_{2}, \ldots, e_{k}$ subject to the usual relations. Then $\operatorname{Pin}(k)$ is defined as the group generated by the $(k-1)$-sphere $S^{k-1}$ consisting of all elements $\sum_{1}^{k} a_{i} e_{i}$ with $\sum_{1}^{k} a_{i}^{2}=1$, the $a_{i}$ being real. Let $p$ denote the canonical projection homomorphism from $\operatorname{Pin}(k)$ onto $O(k)$. Then the kernel of $p$ is $( \pm 1)$, and $\operatorname{Spin}(k)$ is defined as $p^{-1}(S O(k))$. Each element $u \in S^{k-1}$ is mapped by $p$ to a reflection in the hyperplane (in the Euclidean space of which the $e_{i}$ are a basis) perpendicular to $u$. It follows that $\operatorname{Spin}(k)$ cannot contain any product of an odd number of elements of $S^{k-1}$. The centres of the Spin groups may now be found by straightforward algebra. They are as follows.

$$
\begin{aligned}
Z \operatorname{Spin}(2 n+1) & =\mathbb{Z}_{2}, & & n \geqq 1, \\
Z \operatorname{Spin}(4 n+2) & =\mathbb{Z}_{4}, & & n \geqq 1, \\
Z \operatorname{Spin}(4 n) & =\mathbb{Z}_{2} \times \mathbb{Z}_{2}, & & n \geqq 1 .
\end{aligned}
$$

In every case, the centre contains \pm 1 . In the second and third cases, it also contains $4 n+2 \quad 4 n$

$\prod_{1} e_{i}$ or $\prod_{1}^{4 n} e_{i}$ respectively. Now let us consider $\operatorname{Spin}(4 n)$ more closely. The centre, $\mathbb{Z}_{2}^{1} \times \mathbb{Z}_{2}$, contains three distinct $\mathbb{Z}_{2}$ subgroups: the two obvious ones (which we can label as $\mathbb{Z}_{2}^{a}$ and $\mathbb{Z}_{2}^{b}$ ) and the diagonal subgroup, $\mathbb{Z}_{2}^{d}$. If we take $\mathbb{Z}_{2}^{a}$ to be generated by $\prod_{1}^{4 n} e_{i}$, and $\mathbb{Z}_{2}^{b}$ to be generated by $-\prod_{1}^{4 n} e_{i}$, then $\mathbb{Z}_{2}^{d}$ is generated by $\left(\prod_{1}^{4 n} e_{i}\right) \cdot\left(-\prod_{1}^{4 n} e_{i}\right)$ $=-1$, and so $\operatorname{Spin}(4 n) / \mathbb{Z}_{2}^{d}$ is $S O(4 n)$. The question now is whether $\operatorname{Spin}(4 n) / \mathbb{Z}_{2}^{a}$ and $\operatorname{Spin}(4 n) / \mathbb{Z}_{2}^{b}$ are also isomorphic to $S O(4 n)$.

A general result in Lie theory (Helgason, 1978) states the following. Let $G$ be a connected Lie group, and let $A, B$ be distinct subgroups of $Z G$. Then $G / A$ is isomorphic to $G / B$ if and only if there exists an automorphism $\beta: G \rightarrow G$ such that $\beta$ maps $A$ onto $B$. Such an automorphism must be outer, since it has a non-trivial effect on the centre. Now the outer automorphism group of a simply connected 
compact simple Lie group is isomorphic to the symmetry group of the corresponding Dynkin diagram (Wolf, 1967). In the case of Spin(4n), it is therefore clear that the outer automorphism group is $\mathbb{Z}_{2}$ for all $n \geqq 3$. It follows that, modulo inner automorphisms, Spin( $4 n)$ has a unique outer automorphism. All that remains now is to find a explicit representative and to examine its effect on the centre. For any $i, \operatorname{Ad}\left(e_{i}\right)$ is such a representative; for we have, if $u \in \operatorname{Spin}(4 n)$, $\operatorname{det}\left[p\left(\operatorname{Ad}\left(e_{i}\right) u\right)\right]=\operatorname{det}\left[\operatorname{Ad}\left(p e_{i}\right) p u\right]=\operatorname{det}[p u]=1$, so that $\operatorname{Ad}\left(e_{i}\right) u \in \operatorname{Spin}(4 n)$, and so $\operatorname{Ad}\left(e_{i}\right)$ is an automorphism of $\operatorname{Spin}(4 n)$. Evidently it is an outer automorphism. A simple calculation shows that

$$
\operatorname{Ad}\left(e_{i}\right) \prod_{1}^{4 n} e_{j}=-\prod_{1}^{4 n} e_{j}
$$

and so we see that $\operatorname{Spin}(4 n)$ has an automorphism which exchanges $\mathbb{Z}_{2}^{a}$ and $\mathbb{Z}_{2}^{b}$. When $n \geqq 3$, however, there is no other automorphism; and since $\operatorname{Ad}\left(e_{i}\right)$ has no effect on $\mathbb{Z}_{2}^{d}$, we conclude that $\operatorname{Spin}(4 n) / \mathbb{Z}_{2}^{a}$ and $\operatorname{Spin}(4 n) / \mathbb{Z}_{2}^{b}$ are isomorphic to each other, but not to $S O(4 n)$. (In the case of $\operatorname{Spin}(8)$, there is another outer automorphism - triality - and so $\operatorname{Spin}(8) / \mathbb{Z}_{2}^{a}$ and $\operatorname{Spin}(8) / \mathbb{Z}_{2}^{b}$ are in fact isomorphic to $S O(8)$.) Thus, there are two distinct connected groups locally isomorphic to $\operatorname{Spin}(4 n)$ and with centres isomorphic to $\mathbb{Z}_{2}: S O(4 n)$ and $\operatorname{Spin}(4 n) / \mathbb{Z}_{2}, n \geqq 3$.

Now it so happens that the $L(S O(16))$ subalgebra of $L E_{8}$ exponentiates to a $\operatorname{Spin}(16) / \mathbb{Z}_{2}$ - and not to an $S O(16)$ - subgroup of $E_{8}$. The subgroups of $\operatorname{Spin}(16) / \mathbb{Z}_{2}$ are therefore subgroups of $E_{8}$, and so they are of interest to us. For example, $\left\{e_{1}, \ldots, e_{16}\right\}$ can be partitioned into two sets, $\left\{e_{1}, \ldots, e_{6}\right\}$ and $\left\{e_{7}, \ldots, e_{16}\right\}$, generating Spin(6) and Spin(10) subgroups of Spin(16). These correspond, of course, to the $S O(6) \times S O(10)$ subgroup of $S O(16)$. But $S p i n(16)$ does not contain $\operatorname{Spin}(6) \times \operatorname{Spin}(10)$, because these subgroups intersect in $( \pm 1)$. Hence $\operatorname{Spin}(16)$ actually contains $[\operatorname{Spin}(6) \times \operatorname{Spin}(10)] / \mathbb{Z}_{2}$. When $\operatorname{Spin}(16)$ is factored by $\mathbb{Z}_{2}^{d}$, this subgroup projects to $S O(6) \times S O(10)$ in $S O(16)$. But when $\operatorname{Spin}(16)$ is factored by $\mathbb{Z}_{2}^{a}$, the outcome is quite different. Notice that $\operatorname{Spin}(6)$ and $\operatorname{Spin}(10)$ both have centres isomorphic to $\mathbb{Z}_{4}$, generated respectively by $\prod_{1}^{6} e_{i}$ and $\prod_{7}^{16} e_{i}$. On the other hand, $\mathbb{Z}_{2}^{a}$ is generated, by definition, by $\prod_{1}^{16} e_{i}$. Now a simple calculation shows that

$$
\begin{aligned}
& \left(\prod_{1}^{6} e_{i}\right) \cdot\left(\prod_{1}^{16} e_{j}\right)=-\prod_{7}^{16} e_{j} \in \operatorname{Spin}(10), \\
& \left(\prod_{7}^{16} e_{i}\right) \cdot\left(\prod_{1}^{16} e_{j}\right)=-\prod_{1}^{16} e_{j} \in \operatorname{Spin}(6) .
\end{aligned}
$$

In other words, the centres of $\operatorname{Spin}(6)$ and $\operatorname{Spin}(10)$ are identical modulo $\mathbb{Z}_{2}^{a}$. Hence the $[\operatorname{Spin}(6) \times \operatorname{Spin}(10)] / \mathbb{Z}_{2}$ subgroup of $\operatorname{Spin}(16)$ must project to a subgroup of $\operatorname{Spin}(16) / \mathbb{Z}_{2}$ which is isomorphic to $[\operatorname{Spin}(6) \times \operatorname{Spin}(10)] / \mathbb{Z}_{4}$. The centre of this subgroup is $\mathbb{Z}_{4}$.

The situation of $[\operatorname{Spin}(5) \times \operatorname{Spin}(11)] / \mathbb{Z}_{2}$ in $\operatorname{Spin}(16)$ is somewhat different, because whereas $[\operatorname{Spin}(6) \times \operatorname{Spin}(10)] / \mathbb{Z}_{2}$ contains $\prod_{1}^{16} e_{i},[\operatorname{Spin}(5) \times \operatorname{Spin}(11)] / \mathbb{Z}_{2}$ does not. $\left(\operatorname{Spin}(5) \text { does not, of course, contain } \prod_{1}^{5} e_{i}\right)^{1}$ Hence we can write

$$
\mathbb{Z}_{2}^{a} \times[\operatorname{Spin}(5) \times \operatorname{Spin}(11)] / \mathbb{Z}_{2} \subset \operatorname{Spin}(16),
$$


and so when we factor throughout by $\mathbb{Z}_{2}^{a}$, the result is simply [Spin(5) $\times \operatorname{Spin}(11)] / \mathbb{Z}_{2} \subset \operatorname{Spin}(16) / \mathbb{Z}_{2}$; in short, this subgroup of $\operatorname{Spin}(16) / \mathbb{Z}_{2}$ is globally isomorphic to its counterpart in $\operatorname{Spin}(16)$.

It is now possible to compute the centraliser of $\operatorname{Spin}(k), 3 \leqq k \leqq 14$, in $E_{8}$. The strategy is to use Theorem (3.5), with $K=\operatorname{Spin}(16) / \mathbb{Z}_{2}$. Clearly $\operatorname{Spin}(k)$ is a subgroup of $\operatorname{Spin}(16) / \mathbb{Z}_{2}$, so that condition (i) is satisfied. Condition (iii) is satisfied because the algebra of $\operatorname{Spin}(16) / \mathbb{Z}_{2}$ is maximal in that of $E_{8}$, and $Z\left(\operatorname{Spin}(16) / \mathbb{Z}_{2}\right)$ $=\mathbb{Z}_{2}, Z E_{8}=\mathbb{Z}_{1}$. Finally, let us verify condition (ii). The centre of $\operatorname{Spin}(16) / \mathbb{Z}_{2}$ is just the centre of $\operatorname{Spin}(16)$ modulo $\prod_{1}^{16} e_{i}$. The centre of $\operatorname{Spin}(16)$ consists of $\{ \pm 1$, $\left.\pm \prod_{1}^{16} e_{i}\right\}$, and so the centre of $\operatorname{Spin}(16) / \mathbb{Z}_{2}$ is essentially just $\{ \pm 1\}$. This is contained in the centre of every $\operatorname{Spin}(k)$, whether the centre be $\mathbb{Z}_{2}, \mathbb{Z}_{4}$, or $\mathbb{Z}_{2} \times \mathbb{Z}_{2}$. Theorem (3.5) now allows us to compute the $E_{8}$ centraliser of $\operatorname{Spin}(k)$ by computing it in $\operatorname{Spin}(16) / \mathbb{Z}_{2}$ instead. This is a straightforward Clifford algebra computation. One point deserves emphasis. The following group inclusions all correspond to the algebra inclusion $L(S O(6)) \oplus L(S O(10)) \subset L(S O(16))$ :

$$
\begin{gathered}
S O(6) \times S O(10) \subset S O(16), \\
{[\operatorname{Spin}(6) \times \operatorname{Spin}(10)] / \mathbb{Z}_{2} \subset \operatorname{Spin}(16),} \\
{[\operatorname{Spin}(6) \times \operatorname{Spin}(10)] / \mathbb{Z}_{4} \subset \operatorname{Spin}(16) / \mathbb{Z}_{2} .}
\end{gathered}
$$

Now the centraliser of $S O(6)$ in $S O(16)$ is not $S O(10)$. Instead it is $\mathbb{Z}_{2} \times S O(10)$, where $\mathbb{Z}_{2}$ is the centre of $S O(6)$; the centraliser is disconnected. Similarly, the centraliser of $\operatorname{Spin}(6)$ in $\operatorname{Spin}(16)$ is not $\operatorname{Spin}(10)$, but rather the disconnected group $\left[\mathbb{Z}_{4}\right.$ $\times \operatorname{Spin}(10)] / \mathbb{Z}_{2}$. But the centraliser of $\operatorname{Spin}(6)$ in $\operatorname{Spin}(16) / \mathbb{Z}_{2}$ is $\left[\mathbb{Z}_{4} \times \operatorname{Spin}(10)\right] / \mathbb{Z}_{4}$, and this is precisely $\operatorname{Spin}(10)$. Of the three cases, this is the only one in which the centraliser is connected. Similar remarks apply to $\operatorname{Spin}(7)$ and $\operatorname{Spin}(8)$ : the centralisers in $\operatorname{Spin}(16) / \mathbb{Z}_{2}$ - and hence in $E_{8}$-are, respectively, $\operatorname{Spin}(9)$ and $\operatorname{Spin}(8)$ precisely.

We conclude this section with two remarks. According to Theorems (6.1) and (6.2), $S O(6)$ is a candidate holonomy group for a compact Ricci-flat manifold. We therefore wish to embed $S O(6)$ in $E_{8}$ and compute its centraliser (Green et al., 1987). It must be stressed that the considerations of this section give us no information whatever on this problem. The algebra embedding

$$
L(S O(6)) \rightarrow L(S O(16)) \rightarrow L E_{8}
$$

does not exponentiate to an embedding of $S O(6)$ in $E_{8}$, but rather to an embedding of Spin(6); and no six-dimensional manifold can have $\operatorname{Spin}(6)$ as its linear holonomy group. As we shall see, $S O(6)$ can be embedded in $E_{8}$ in a different way, but then its centraliser is not $S O(10)$, even locally. In a word, it is not correct to assert that string compactification on a manifold with $S O(6)$ holonomy leads to $S O(10)$ grand unification. (Of course, one could try to use the spinor holonomy bundle instead of the linear holonomy bundle; but then this would have to be done consistently, i.e. the consequences for $S U(3)$ "holonomy" would require investigation. These and other unorthodox interpretations of the formalism will be considered elsewhere.)

Secondly and finally, a technical remark: according to the above, the centraliser of $\operatorname{Spin}(3)$ in $E_{8}$ is $\operatorname{Spin}(13)$. But $\operatorname{Spin}(3)=S U(2)$, and we claimed in Sect. 4 that 
$C(S U(2))=E_{7}$. The problem here is that we are dealing with two different embeddings of $S U(2)$ in $\operatorname{Spin}(16) / \mathbb{Z}_{2}$. Very briefly, we have

$$
\operatorname{Spin}(3) \rightarrow \operatorname{Spin}(4) \rightarrow \operatorname{Spin}(16) / \mathbb{Z}_{2} \text {. }
$$

Now $\operatorname{Spin}(4)=S U(2) \times S U(2)$. The above $\operatorname{Spin}(3)$ corresponds to the diagonal subgroup, and it contains the centre of $\operatorname{Spin}(16) / \mathbb{Z}_{2}$ (so that Theorem (3.5) applies). But if we choose to embed $S U(2)$ through an explicit $S U(2)$ factor in Spin(4), then this $S U(2)$ does not contain the centre of $\operatorname{Spin}(16) / \mathbb{Z}_{2}$, and so the $E_{8}$ centraliser can no longer be computed in $\operatorname{Spin}(16) / \mathbb{Z}_{2}$. Instead we note that since $S U(2) \subset \operatorname{Spin}(4)$ and $C \operatorname{Spin}(4)=\operatorname{Spin}(12)$, clearly $\operatorname{Spin}(12) \subset C(S U(2))$. In fact $[S U(2) \times \operatorname{Spin}(12)] / \mathbb{Z}_{2}$ $C C(S U(2))$, and this alone eliminates all possibilities save $E_{7}$. (See Slansky's (1981) list of maximal subalgebras of the exceptional algebras.)

The main result of this section can be stated as follows.

Theorem (7.1) Let $\operatorname{Spin}(k), 3 \leqq k \leqq 14$, be embedded in $E_{8}$ through the [Spin $(k)$ $\times \operatorname{Spin}(16-k)] / Z \operatorname{Spin}(k)$ subgroup of $\operatorname{Spin}(16) / \mathbb{Z}_{2}$. Then the full centraliser of $\operatorname{Spin}(k)$ in $E_{8}$ is $\operatorname{Spin}(16-k)$.

\section{Some Disconnected Subgroups of $E_{8}$}

We are now in a position to embed, and compute the centralisers of, many of the groups listed in Theorems (6.1) and (6.2). This includes several of the disconnected holonomy groups. For example, let $M$ be a compact 8-dimensional manifold such that the identity component of the holonomy group is $S p(2)$, and suppose that $M$ is not simply connected. According to Theorem (6.2), the full holonomy group must be $\mathbb{Z}_{3} \times S p(2)$. Now $S p(2)$ is globally isomorphic to $\operatorname{Spin}(5)$ (Curtis, 1984). Hence $S p(2)$ has a natural embedding in $E_{8}$, through $\operatorname{Spin}(16) / \mathbb{Z}_{2}$, and its centraliser is $\operatorname{Spin}(11)$. Now the $\mathbb{Z}_{3}$ factor in the holonomy group commutes with $S p(2)$, and so it must be embedded in $\operatorname{Spin}(11)$. One way to do this is to embed $\mathbb{Z}_{3}$ in $U(1)$ and then to note that $\operatorname{Spin}(11)$ contains $[U(1) \times \operatorname{Spin}(9)] / \mathbb{Z}_{2}$, since $U(1)=\operatorname{Spin}(2)$. The centraliser of $\mathbb{Z}_{3} \times S p(2)$ is then $[U(1) \times \operatorname{Spin}(9)] / \mathbb{Z}_{2}$.

This strategy fails, however, when the product is semidirect rather than direct. For example, we cannot compute the centraliser of $S U(3) \rtimes \mathbb{Z}_{2}$ [see Theorem (6.2)] in $E_{8}$ by embedding $\mathbb{Z}_{2}$ in $E_{6}$, precisely because $\mathbb{Z}_{2}$ does not centralise every element of $S U(3)$. We must find an element $\zeta$ in $E_{8}$ which is contained neither in $S U(3)$ nor in $E_{6}$, such that $\zeta^{2}=1$ and such that $\operatorname{Ad}(\zeta)$ maps each $S \in S U(3)$ to its complex conjugate. As we have no explicit way of presenting $E_{8}$, this is not a simple problem. Furthermore, we need to solve the analogous problems for $S U(4), S U(5)$ and so on. Of course, it is entirely possible a priori that $S U(3) \rtimes \mathbb{Z}_{2}, S U(4) \rtimes \mathbb{Z}_{2}$ and so on simply cannot be embedded in $E_{8}$ in any way whatever; after all, there is no fundamental relationship between holonomy theory and the structure of $E_{8}$. It is therefore remarkable that, in fact, $S U(3) \rtimes \mathbb{Z}_{2}, S U(4)$ $\rtimes \mathbb{Z}_{2}$, and $S U(5) \rtimes \mathbb{Z}_{2}$ all have natural embeddings in $E_{8}$.

Recall that $S U(2), S U(3)$, and $S U(5)$ all have canonical embeddings in $E_{8}$ through the subgroups $S U(2) \cdot E_{7}, S U(3) \cdot E_{6}$, and $S U(5) \cdot S U(5)$. (See Sect. 6 for the dot notation.) Thus far we have not mentioned $S U(4)$. It is globally isomorphic to $\operatorname{Spin}(6)$, and so the natural embedding in $E_{8}$ is through $\operatorname{Spin}(16) / \mathbb{Z}_{2}$ :

$$
S U(4)=\operatorname{Spin}(6) \rightarrow[\operatorname{Spin}(6) \times \operatorname{Spin}(10)] / \mathbb{Z}_{4} \rightarrow \operatorname{Spin}(16) / \mathbb{Z}_{2} \rightarrow E_{8} .
$$


By Theorem (7.1), the centraliser of $S U(4)$ in $E_{8}$ is precisely $\operatorname{Spin}(10)$. We call this the canonical embedding of $S U$ (4). It is now possible to establish embeddings of $S U(3) \rtimes \mathbb{Z}_{2}, S U(4) \rtimes \mathbb{Z}_{2}$, and $S U(5) \rtimes \mathbb{Z}_{2}$.

Theorem (8.1) $E_{8}$ has subgroups of the form $S U(3) \rtimes \mathbb{Z}_{2}, S U(4) \rtimes \mathbb{Z}_{2}$, and $S U(5)$ $\rtimes \mathbb{Z}_{2}$, where $S U(3), S U(4)$, and $S U(5)$ are embedded canonically, and where $\mathbb{Z}_{2}$ acts through complex conjugation. The centralisers in $E_{8}$ are

$$
\begin{aligned}
& C\left[S U(3) \rtimes \mathbb{Z}_{2}\right]=S p(4) / \mathbb{Z}_{2}, \\
& C\left[S U(4) \rtimes \mathbb{Z}_{2}\right]=[\operatorname{Spin}(5) \times \operatorname{Spin}(5)] / \mathbb{Z}_{2}, \\
& C\left[S U(5) \rtimes \mathbb{Z}_{2}\right]=S O(5) .
\end{aligned}
$$

Proof. The strategy is as follows. First we find an element $\zeta_{0}$ in $E_{8}$ such that $\zeta_{0} z \zeta_{0}^{-1}$ $=z^{-1}$, where $z$ generates the centre of the $\operatorname{Spin}(10)$ subgroup of $E_{8}$. Then we show that $\operatorname{Ad}\left(\zeta_{0}\right)$ induces an outer automorphism on $\operatorname{Spin}(10)$. This leads us to an element $\zeta \in E_{8}$ such that $\operatorname{Ad}(\zeta)$ induces complex conjugation on $S U(3), S U(4)$, and $S U(5)$. After showing that $\zeta^{2}=1$, we find the above centralisers by studying the fixed point sets of $\operatorname{Ad}(\zeta)$ in $E_{6}$, Spin(10), and $S U(5)$.

Let $R$ be an irreducible root system in a finite-dimensional real vector space $V$, and let $I_{v}$ be the identity automorphism of $V$. By the definition of $R,-I_{v}$ is an automorphism of $R$. It can be shown (Humphreys, 1972) that the full automorphism group of $R$ is isomorphic to the semi-direct product of $W(R)$, the Weyl group of $R$, with $D(R)$, the group of automorphisms of the corresponding Dynkin diagram. In the case of the root system of $E_{8}, D(R)$ is obviously trivial, and so $W(R)$ contains $-I_{v}$. In other words, the Weyl group of $E_{8}$ contains an element which simultaneously reverses the sign of every real root.

At the group level, this means the following. Let $\alpha$ be a real root of $E_{8}$; then the corresponding global root (Bröcker and tomDieck, 1985) is the homomorphism given by $\exp (H) \rightarrow \exp (2 \pi i \alpha(H))$, where $\exp (H) \in T$, a maximal torus. Now at the group level, the presence of $-I_{v}$ in the Weyl group of the $E_{8}$ root system just means that there is an element of the (global) Weyl group which maps each $\exp (H)$ to $\exp (-H)$, since every real root has its sign reversed. On the other hand, the action of the Weyl group on $T$ is, by definition, the same as that of the normaliser $N(T)$ of $T$ in $E_{8}$.

Now let $z$ be a fixed generator of the centre of the canonical Spin(10) subgroup of $E_{8}$. (Recall that this centre is $\mathbb{Z}_{4}$, so it is generated by a single element.) Since $E_{8}$ is compact and connected, it is covered by its maximal tori. Let $T$ be a maximal torus of $E_{8}$ containing $z$. According to the above discussion, $N(T)$ must contain an element $\zeta_{0}$ such that

$$
\operatorname{Ad}\left(\zeta_{0}\right) z=\zeta_{0} z \zeta_{0}^{-1}=z^{-1} .
$$

Notice that we have reached this conclusion using only the fact that the Dynkin diagram of $E_{8}$ has no symmetries. A similar argument therefore works for all of the exceptional groups except $E_{6}$.

Now let $s$ be any element of the $\operatorname{Spin}(6) \cdot \operatorname{Spin}(10)$ subgroup of $E_{8}$. Clearly, $\operatorname{Ad}\left(\zeta_{0}^{2}\right) z=z$, so $\operatorname{Ad}\left(\zeta_{0}^{-1}\right) z=z^{-1}$; that is, $z \zeta_{0}=\zeta_{0} z^{-1}$. Therefore

$$
\operatorname{Ad}(z) \operatorname{Ad}\left(\zeta_{0}\right) s=\operatorname{Ad}\left(z \zeta_{0}\right) s=\operatorname{Ad}\left(\zeta_{0} z^{-1}\right) s=\operatorname{Ad}\left(\zeta_{0}\right) s
$$

because $z$ generates the common centre of $\operatorname{Spin}(6)$ and $\operatorname{Spin}(10)$. Hence $\operatorname{Ad}\left(\zeta_{0}\right) s \in C \mathbb{Z}_{4}$, where $C$ denotes the centraliser in $E_{8}$. Since $s$ is arbitrary and since $\operatorname{Ad}\left(\zeta_{0}\right)$ is a continuous map, we have 


$$
\operatorname{Ad}\left(\zeta_{0}\right): \operatorname{Spin}(6) \cdot \operatorname{Spin}(10) \rightarrow C_{0} \mathbb{Z}_{4},
$$

the identity component of $C \mathbb{Z}_{4}$. Now in the proof of Theorem (3.5), we saw that any connected subgroup $K \subset G$ which satisfies condition (iii) of that theorem also satisfies $K=C_{0} Z K$. As $\operatorname{Spin}(6) \cdot \operatorname{Spin}(10)$ does satisfy this condition, we have $C_{0}\left(\mathbb{Z}_{4}\right)=\operatorname{Spin}(6) \cdot \operatorname{Spin}(10)$ and so we see that $\operatorname{Ad}\left(\zeta_{0}\right)$ is an automorphism of $\operatorname{Spin}(6) \cdot \operatorname{Spin}(10)$. As it has a non-trivial effect on the centre, it is an outer automorphism. The following lemma allows us to study the restriction of this automorphism to $\operatorname{Spin}(10)$.

Lemma (8.2). Let $G$ be a compact, connected Lie group and let $K$ be a connected, simple subgroup of $G$. Assume that rank $K>\frac{1}{2} \operatorname{rank} G$. Then any automorphism of $K \cdot C K$ restricts to an automorphism of $K$.

Proof. Evidently $Z K$ is a normal subgroup of $C K$. Let $\lambda$ be the projection $\lambda: C K$ $\rightarrow C K / Z K$. Given any automorphism $\phi: K \cdot C K \rightarrow K \cdot C K$, define a homomorphism $\hat{\phi}: K \rightarrow C K / Z K$ as follows. If $k \in K$, set $\phi(k)=x y$, where $x \in K, y \in C K$. Then define

$$
\hat{\phi}(k)=\lambda(y) .
$$

This is well-defined, because if $x y=\bar{x} \bar{y}$ for some other pair $\bar{x}, \bar{y}$, then $\bar{y} y^{-1} \in K \cap C K$ $=Z K$, and therefore $\lambda(y)=\lambda(\bar{y})$. Furthermore, $\hat{\phi}$ is a homomorphism, for if $\phi\left(k_{1}\right)$ $=x_{1} y_{1}$ and $\phi\left(k_{2}\right)=x_{2} y_{2}$, then $\phi\left(k_{1} k_{2}\right)=x_{1} x_{2} y_{1} y_{2}$ and so $\hat{\phi}\left(k_{1} k_{2}\right)=\lambda\left(y_{1} y_{2}\right)$ $=\hat{\phi}\left(k_{1}\right) \hat{\phi}\left(k_{2}\right)$. It therefore follows that the image $\hat{\phi}(K)$ of $K$ in $C K / Z K$ is isomorphic to $K / N$ for some normal subgroup $N$ in $K$. Since $K$ is connected and simple, we have either $N=K$ or $N$ is discrete. Assume the latter. Then $K / N \subseteq C K / Z K$ implies rank $K \leqq$ rank $C K$, because $Z K$ must also be discrete if $K$ is simple. On the other hand, $K \cdot C K \subseteq G$ implies rank $K+\operatorname{rank} C K-\operatorname{rank}(K \cap C K)$ $\leqq \operatorname{rank} G$. But $\operatorname{rank}(K \cap C K)=\operatorname{rank} Z K=0$, and so $2 \operatorname{rank} K \leqq \operatorname{rank} G$, contrary to our assumption that rank $K>\frac{1}{2} \operatorname{rank} G$. Therefore $N$ is not discrete and we must have $N=K$. Thus $\hat{\phi}$ maps all of $K$ to the identity in $C K / Z K$, and so when we write $\phi(k)=x y$, it must be the case that $y \in Z K$ and so $y \in K$. Thus $\phi(k) \in K$, and this completes the proof of the Lemma.

Returning to the proof of the Theorem, we choose $K=\operatorname{Spin}(10), G=E_{8}$, so that $C K=\operatorname{Spin}(6)$. Then $K$ is connected and simple, and rank $K=5$ while $\frac{1}{2}$ rank $E_{8}=4$. Therefore $\operatorname{Ad}\left(\zeta_{0}\right)$ restricts to an outer automorphism of Spin(10). Now the Dynkin diagram of Spin(10) has a single symmetry, and so, modulo inner automorphisms, $\operatorname{Spin}(10)$ has a unique outer automorphism. Using the same argument as in Sect. 7, one shows that $\operatorname{Ad}(\theta)$, where $\theta=e_{1} e_{2} e_{3} e_{4} e_{5}$, represents this outer automorphism. By uniqueness, it follows that there exists $s \in \operatorname{Spin}(10)$ such that $\operatorname{Ad}\left(\zeta_{0}\right) \circ \operatorname{Ad}(s)$ $=\operatorname{Ad}(\theta)$. If we define $\zeta_{0} s=\zeta$, then we have found $\zeta \in E_{8}$ such that

$$
\operatorname{Ad}(\zeta)=\operatorname{Ad}(\theta)
$$

on Spin(10). (Of course, $\theta$ is an element of Pin(10), not of $E_{8}$.)

Now $S U(5)$ embeds in $S O(10)$ through the real representation:

$$
A+i B \rightarrow\left[\begin{array}{cc}
A & -B \\
B & A
\end{array}\right],
$$


where $A$ and $B$ are real $5 \times 5$ matrices such that $A+i B \in S U(5)$. If $p: \operatorname{Spin}(10)$ $\rightarrow S O(10)$ is the projection, consider $p^{-1}[S U(5)]$. This subgroup of $\operatorname{Spin}(10)$ cannot be connected; for if we assume the contrary, then $p^{-1}(S U(5))$ would be a connected group locally isomorphic to $S U(5)$, with $\mathbb{Z}_{2}$ as a normal subgroup. By continuity, a discrete normal subgroup must be central, but no connected group locally isomorphic to $S U(5)$ has a $\mathbb{Z}_{2}$ in its centre. Since this $\mathbb{Z}_{2}$ is central in Spin(10), the only possibility is that $p^{-1}(S U(5))$ is isomorphic to $\mathbb{Z}_{2} \times S U(5)^{a}$, where the superscript merely indicates that $S U(5)^{a}$ is contained in $\operatorname{Spin}(10)$ rather than SO(10).

"Complex conjugation" on the $S U(5)$ subgroup of $S O(10)$ is defined so that it commutes with the embedding homomorphism - that is, by

$$
\left(\begin{array}{cc}
A & -B \\
B & A
\end{array}\right) \rightarrow\left(\begin{array}{cc}
A & B \\
-B & A
\end{array}\right)
$$

Similarly, complex conjugation on $S U(5)^{a}$ is defined by

$$
s \rightarrow \bar{s}=p_{a}^{-1}[\overline{p s}],
$$

where $p_{a}$ denotes the restriction of $p$ to $S U(5)^{a}$. Now let $s \in S U(5)^{a}$. Then

$$
p \operatorname{Ad}(\zeta) s=p \operatorname{Ad}(\theta) s=\operatorname{Ad}(p \theta) p s .
$$

Recall that for each $e_{i}, p\left(e_{i}\right)$ is a reflection in the plane perpendicular to $e_{i}$. Therefore $p\left(e_{1} e_{2} e_{3} e_{4} e_{5}\right)$ corresponds to the $O(10)$ matrix $\left[\begin{array}{cc}-I_{5} & 0 \\ 0 & I_{5}\end{array}\right]$. A simple calculation shows that if $p s \in S U(5)$ is $\left[\begin{array}{cc}A & -B \\ B & A\end{array}\right]$, then $\operatorname{Ad}(p \theta) p s$ is $\left[\begin{array}{cc}A & B \\ -B & A\end{array}\right]=\overline{p s}$, which is also an element of $S U(5)$. Hence $p \operatorname{Ad}(\zeta) s \in S U(5)$ for all $s \in S U(5)^{a}$; that is, $\operatorname{Ad}(\zeta): S U(5)^{a} \rightarrow p^{-1} S U(5)$. By continuity, $\operatorname{Ad}(\zeta): S U(5)^{a} \rightarrow S U(5)^{a}$. Hence $p \operatorname{Ad}(\zeta) s$ $=p_{a} \operatorname{Ad}(\zeta) s=\operatorname{Ad}(p \theta) p s=\overline{p s}$, and so

$$
\operatorname{Ad}(\zeta) s=p^{-1}(\bar{p} \bar{s})=\bar{s}
$$

That is, the restriction of $\operatorname{Ad}(\zeta)$ to $S U(5)^{a}$ induces complex conjugation.

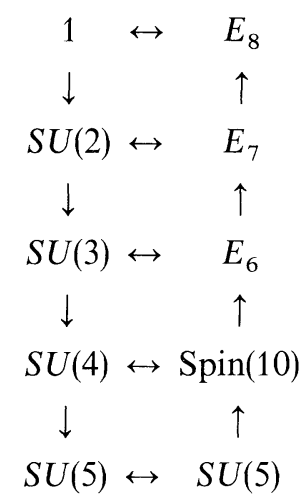

Consider the diagram. The vertical arrows denote inclusions, while the horizontal arrows denote the centraliser. Either $S U(5)$ in $S U(5) \cdot S U(5)$ may be identified with $S U(5)^{a}$, and so $\operatorname{Ad}(\zeta)$ induces complex conjugation on both. Hence it induces complex conjugation on the canonical $S U(2), S U(3)$, and $S U(4)$ subgroups of $E_{8}$. 
Next we show that $\zeta^{2}=1$. On $\operatorname{Spin}(10), \operatorname{Ad}(\zeta)=\operatorname{Ad}(\theta)$. $\operatorname{But} \theta^{2}=-1$, so $\operatorname{Ad}\left(\zeta^{2}\right)$ is the identity automorphism on $\operatorname{Spin}(10)$; that is, $\zeta^{2}$ centralises $\operatorname{Spin}(10)$. Therefore $\zeta^{2} \in S U(4)$. But since $\operatorname{Ad}(\zeta)$ induces complex conjugation on $S U(4), \zeta^{2}$ also centralises $S U(4)$, and so $\zeta^{2} \in S U(4) \cap \operatorname{Spin}(10)=\mathbb{Z}_{4}$. Thus $\zeta^{8}=1$. Applying the same argument to $S U(5) \cdot S U(5)$, we obtain $\zeta^{10}=1$. Therefore $\zeta^{2}=1$.

Obviously $\zeta$ cannot be an element of $S U(3), S U(4)$, or $S U(5)$. The groups $S U(m)$ $\cup \zeta \cdot S U(m), m=3,4,5$, are therefore subgroups of $E_{8}$ with structure $S U(m) \rtimes \mathbb{Z}_{2}$. This completes the first part of the proof.

We now compute the centralisers. The centraliser of $S U(5)$ is just (the other) $S U(5)$. Therefore the centraliser of $S U(5) \rtimes \mathbb{Z}_{2}$ is just the fixed point set of $\operatorname{Ad}(\zeta)$ in $S U(5)$. As $\operatorname{Ad}(\zeta)$ acts on $S U(5)$ by complex conjugation, the fixed point set is the real subgroup of $S U(5)$, namely $S O(5)$. Hence $C\left[S U(5) \rtimes \mathbb{Z}_{2}\right]=S O(5)$. Similarly, $C[S U(4)]=\operatorname{Spin}(10)$, and $\operatorname{Ad}(\zeta)$ acts through $\operatorname{Ad}(\theta)$. Since $\theta=e_{1} e_{2} e_{3} e_{4} e_{5}$, an easy calculation shows that the fixed point set is $[\operatorname{Spin}(5) \times \operatorname{Spin}(5)] / \mathbb{Z}_{2}$. Notice that if we embed $\operatorname{Spin}(5)$ in $\operatorname{Spin}(5) \times \operatorname{Spin}(5)$ diagonally, then

$$
\begin{aligned}
C\left[S U(5) \rtimes \mathbb{Z}_{2}\right) & =S O(5)=\operatorname{Spin}(5) / \mathbb{Z}_{2} \subset[\operatorname{Spin}(5) \times \operatorname{Spin}(5)] / \mathbb{Z}_{2} \\
& =C\left[S U(4) \rtimes \mathbb{Z}_{2}\right]
\end{aligned}
$$

in agreement with the fact that $S U(4) \rtimes \mathbb{Z}_{2}$ is a subgroup of $S U(5) \rtimes \mathbb{Z}_{2}$.

Finally, $C[S U(3)]=E_{6}$, so we need to find the fixed point set of $\operatorname{Ad}(\zeta)$ in $E_{6}$. Notice first that if an automorphism of a group restricts to an automorphism of a subgroup, then it also restricts to an automorphism of the centraliser of that subgroup. Hence $\operatorname{Ad}(\zeta)$ is an automorphism of $E_{6}$. Since $\zeta^{2}=1$, it is an involutive automorphism. We therefore need information on the involutive outer automorphisms of $E_{6}$. Fortunately, the formidable task of classifying these was undertaken by Wolf (1967), and so we merely need to interpret his results.

Theorem (8.3). There exist two distinguished involutive outer automorphisms, $\psi_{1}$ and $\psi_{2}$, of $E_{6}$. The fixed point sets of these automorphisms are isomorphic respectively to $F_{4}$ and $S p(4) / \mathbb{Z}_{2}$. Every involutive outer automorphism of $E_{6}$ is $\operatorname{Ad}\left(\operatorname{Ad}\left(E_{6}\right)\right)$ conjugate either to $\psi_{1}$ or to $\psi_{2}$.

Proof. Wolf (1967), p. 288.

The meaning of the final statement is as follows: if $\psi$ is an involutive outer automorphism of $E_{6}$, then there exists $g \in E_{6}$ such that $\psi=\operatorname{Ad}(g) \circ \psi_{i} \circ \operatorname{Ad}\left(g^{-1}\right)$ for $i=$ either 1 or 2 . The point is this. Suppose that $\psi=\operatorname{Ad}(g) \circ \psi_{1} \circ \operatorname{Ad}\left(g^{-1}\right)$. Then the fixed point set of $\psi$ is clearly $\operatorname{Ad}(g) F_{4}$, which is another subgroup of $E_{6}$ isomorphic to $F_{4}$. Similarly, $\operatorname{Ad}(g) \circ \psi_{2} \circ \operatorname{Ad}\left(g^{-1}\right)$ has a fixed point set isomorphic to $S p(4) / \mathbb{Z}_{2}$. According to the theorem, then, every involutive outer automorphism of $E_{6}$ has a fixed point set isomorphic either to $F_{4}$ or to $S p(4) / \mathbb{Z}_{2}$.

$\operatorname{As} \operatorname{Ad}(\zeta)$ is an involutive outer automorphism of $E_{6}$, its fixed point set must be isomorphic to either $F_{4}$ or $S p(4) / \mathbb{Z}_{2}$. Now clearly $S U(3) \rtimes \mathbb{Z}_{2} \subset S U(4) \rtimes \mathbb{Z}_{2}$, and so $[\operatorname{Spin}(5) \times \operatorname{Spin}(5)] / \mathbb{Z}_{2}=C\left[S U(4) \rtimes \mathbb{Z}_{2}\right] \subseteq C\left[S U(3) \rtimes \mathbb{Z}_{2}\right]$. But $F_{4}$ contains no such subgroup, as a glance at the list of maximal subalgebras of $L F_{4}$ immediately shows. However, we have $S p(2) \times S p(2) \subset S p(4)$, with the centre of $S p(4)$ diagonal between those of the two $S p(2)$ subgroups; hence $[\operatorname{Spin}(5) \times \operatorname{Spin}(5)] / \mathbb{Z}_{2}=[S p(2)$ $\times S p(2)] / \mathbb{Z}_{2} C S p(4) / \mathbb{Z}_{2}$. Thus $S p(4) / \mathbb{Z}_{2}$ is the only possibility for $C\left[S U(3) \rtimes \mathbb{Z}_{2}\right]$. This completes the proof of Theorem (8.1).

We are now in a position to attack the classification problem. 


\section{The Classification: Gauge Groups in $E_{8}$}

Recall that we are interested in compact, Ricci-flat Riemannian manifolds $M$ such that $\operatorname{dim}(M)>1$ and $M$ is not locally isometric to a product. (We shall say that $M$ is locally irreducible.) The holonomy bundle is extended to an $E_{8}$ bundle. The exterior symmetry group $E(\omega)$ is finite for such $M$, but the interior symmetry group $I(\omega)$ is to be identified with the gauge group of the theory. According to Theorem (2.1), we shall therefore obtain a complete classification of all possible gauge subgroups of $E_{8}$ if we can compute the centraliser of every "holonomy subgroup" of $E_{8}$.

Here we shall not be quite so ambitious. Unfortunately, $C \Psi(M)$ depends on the embedding of $\Psi(M)$ in $E_{8}$. However, it so happens that there is almost always a particularly obvious and natural way of embedding the groups listed in Theorems (6.1) and (6.2) in $E_{8}$. We shall confine attention to these embeddings. Subject to this condition, the classification below is complete; the reader will have no difficulty in adapting our methods to other embeddings.

The possible values of $n=\operatorname{dim} M$ are $4-10,12,14$, and 16. Beyond $n=16$, the holonomy groups are too large to embed in $E_{8}$. We consider each value of $n$ in turn.

$n=4$ : According to Theorems (6.1) and (6.2), the candidates for $\Psi(M)$ are $S O(4)$, $O(4), S p(1), \mathbb{Z}_{4} \cdot S p(1)$, and $Q_{8} \cdot S p(1)$. Of course, $S p(1)$ is isomorphic to $S U(2)$.

We know that the centraliser of $S U(4)$ in $E_{8}$ is $\operatorname{Spin}(10)$. In $S U(4), S O(4)$ is the subgroup fixed by complex conjugation. If $\zeta$ is the element of $E_{8}$ defined in the preceding section, then $S O(4)$ is precisely the subset of $S U(4)$ which consists of elements commuting with $\zeta$. The centraliser of $S O(4)$ in $E_{8}$ is therefore $\operatorname{Spin}(10)$ $\cup \zeta \cdot \operatorname{Spin}(10)$, which is the semi-direct product $\operatorname{Spin}(10) \rtimes \mathbb{Z}_{2}$ (where $\mathbb{Z}_{2}$ acts on $\operatorname{Spin}(10)$ in the same way as $\left.\operatorname{Ad}\left(e_{1} e_{2} e_{3} e_{4} e_{5}\right)\right)$. Thus $C[S O(4)]=\operatorname{Spin}(10) \rtimes \mathbb{Z}_{2}$. (This group is not, incidentally, isomorphic to Pin(10).)

Similarly, $O(4)$ is the subgroup of $U(4)$ fixed by $\operatorname{Ad}(\zeta)$. We can embed $U(4)$ in $S U(5)$ as follows:

$$
u \rightarrow\left[\begin{array}{ll}
u & 0 \\
0 & \delta
\end{array}\right], \quad \delta=[\operatorname{det} u]^{-1} .
$$

Then $U(4)$ embeds in $E_{8}$ through $S U(5) \cdot S U(5)$. The centraliser in $S U(5) \cdot S U(5)$ (and hence - using Theorem (3.5) - in $\left.E_{8}\right)$ is $U(1) \cdot S U(5)=[U(1) \times S U(5)] / \mathbb{Z}_{5}$ $=U(5)$. Arguing as above, we find $C[O(4)]=U(5) \rtimes \mathbb{Z}_{2}$, where $\mathbb{Z}_{2}$ acts as complex conjugation.

$S p(1)=S U(2)$ was treated in Sect. 4. (See also the end of Sect. 7.) We found that $C[S U(2)]=E_{\gamma}$.

In $\mathbb{Z}_{4} \cdot S p(1)$, the $\mathbb{Z}_{4}$ factor centralises $S p(1)$, and so we must embed it in $E_{7}$. The obvious way to proceed is to embed $\mathbb{Z}_{4}$ in the $U(1)$ factor of $U(1) \cdot E_{6} \subset E_{7}$. Notice that the $\mathbb{Z}_{4} \cdot S p(1)$ notation means that $\mathbb{Z}_{4}$ and $S p(1)$ intersect in $\mathbb{Z}_{2}$, so we must verify that the $\mathbb{Z}_{4}$ in $U(1) \cdot E_{6}$ does contain this $\mathbb{Z}_{2}$. That is easy: the $\mathbb{Z}_{2}$ in question is also the centre of $E_{7}$, and so it must be contained in the centre of $U(1) \cdot E_{6}$ because the latter is connected and is of maximal rank in $E_{7}$. The centraliser of $\mathbb{Z}_{4}$ in $E_{7}$ is the same as that of $U(1)$; since $U(1)$ is a torus, its centraliser is connected, and so we find $C\left[\mathbb{Z}_{4} \cdot S p(1)\right]=U(1) \cdot E_{6}=\left[U(1) \times E_{6}\right] / \mathbb{Z}_{3}$.

Finally, the centraliser of $Q_{8} \cdot S p(1)$ can be found most easily by noting that any matrix representation of $Q_{8}$ is centralised precisely by those elements that centralise the corresponding $S U(2)$. But $S U(2) \cdot S U(2)=[S U(2) \times S U(2)] / \mathbb{Z}_{2}$ is precisely $S O(4)$. Thus $C\left[Q_{8} \cdot S p(1)\right]=C[S O(4)]=\operatorname{Spin}(10) \rtimes \mathbb{Z}_{2}$. 
$n=5$ : The possibilities for $\Psi(M)$ are $S O(5)$ and $O(5)$. Using the same method as for $S O(4)$ and $O(4)$, one finds that $C[S O(5)]=S U(5) \rtimes \mathbb{Z}_{2}$ and $C[O(5)]=U(4) \rtimes \mathbb{Z}_{2}$.

$n=6$ : The possibilities for $\Psi(M)$ are $S O(6), O(6), S U(3)$, and $S U(3) \rtimes \mathbb{Z}_{2}$. The latter two have been dealt with at length, and we have also discussed the fact that $S O(6)$ cannot be embedded in $E_{8}$ through the " $S O(16)$ " subgroup. Instead we embed it in the $S U(6)$ subgroup of $E_{7}$, as the real subgroup. The embedding in $E_{8}$ then takes the form

$$
S O(6) \rightarrow S U(6) \rightarrow[S U(2) \times S U(3)] \cdot S U(6) \rightarrow S U(2) \cdot E_{7} \rightarrow E_{8},
$$

where $[S U(2) \times S U(3)] \cdot S U(6)$ denotes $[(S U(2) \times S U(3)) \times S U(6)] / \mathbb{Z}_{6}$. The centre of this group is the common centre $\mathbb{Z}_{6}=\mathbb{Z}_{2} \times \mathbb{Z}_{3}$ of $S U(2) \times S U(3)$ and $S U(6)$. The only connected subgroups of $E_{8}$ which contain $[S U(2) \times S U(3)] \cdot S U(6)$ are $S U(2) \cdot E_{7}, S U(3) \cdot E_{6}$, and $E_{8}$, with respective centres $\mathbb{Z}_{2}, \mathbb{Z}_{3}$, and $\mathbb{Z}_{1}$. Applying Theorem (3.5), we obtain $C(S U(6))=S U(2) \times S U(3)$. We can determine $C(S O(6))$ with the aid of the following lemma.

Lemma (9.1). $E_{8}$ contains a subgroup of the form $\left[S U(9) / \mathbb{Z}_{3}\right] \rtimes \mathbb{Z}_{2}$, where $\varrho$, the generator of $\mathbb{Z}_{2}$, is such that $\operatorname{Ad}(\varrho)$ induces the automorphism of $S U(9) / \mathbb{Z}_{3}$ corresponding to complex conjugation on $S U(9)$.

Proof. We know that $E_{8}$ contains $S U(9) / \mathbb{Z}_{3}$ as a maximal connected subgroup. As in the proof of Theorem (8.1), let $\varrho_{0} \in E_{8}$ be such that $\operatorname{Ad}\left(\varrho_{0}\right) z=z^{-1}$, where $z$ generates the $\mathbb{Z}_{3}$ centre of $S U(9) / \mathbb{Z}_{3}$. Then $\operatorname{Ad}\left(\varrho_{0}\right)$ is an outer automorphism of $S U(9) / \mathbb{Z}_{3}$. Now given any group $G$, an automorphism $\phi$ of $G$, and a normal subgroup $N$ in $G$, one can easily show that $\phi$ induces an automorphism on $G / N$ if and only if $\phi$ restricts to an automorphism of $N$. That is the case for the action of complex conjugation on the $\mathbb{Z}_{3}$ in the centre of $S U(9)$, so complex conjugation induces an outer automorphism on $S U(9) / \mathbb{Z}_{3}$, which we shall denote by $\gamma$. The outer automorphism group of $S U(9)$ being $\mathbb{Z}_{2}$, we see that there exists $s \in S U(9) / \mathbb{Z}_{3}$ such that $\operatorname{Ad}\left(\varrho_{0} S\right)=\gamma$ on $S U(9) / \mathbb{Z}_{3}$. Let $\varrho=\varrho_{0} S$. Now notice that if $K$ is any group satisfying the conditions of Theorem (3.5), then choosing $H=K$ we obtain

$$
C K=C_{K} K=Z K \text {. }
$$

Therefore the $E_{8}$ centraliser of $S U(9) / \mathbb{Z}_{3}$ is $\mathbb{Z}_{3}$. Since $\operatorname{Ad}\left(\varrho^{2}\right)=\gamma^{2}$ is trivial on $S U(9) / \mathbb{Z}_{3}$, it follows that $\varrho^{2} \in \mathbb{Z}_{3}$ so $\varrho^{6}=1$. But $\operatorname{Ad}(\varrho)$ induces complex conjugation on the $S U(5)$ subgroup of $S U(9) / \mathbb{Z}_{3}$, and this $S U(5)$ is actually the canonical one in $E_{8}$. Thus $\varrho^{10}=1$ and so $\varrho^{2}=1$. The group $S U(9) / \mathbb{Z}_{3} \cup \varrho \cdot S U(9) / \mathbb{Z}_{3}$ is therefore isomorphic to $\left[S U(9) / \mathbb{Z}_{3}\right] \times \mathbb{Z}_{2}$. This completes the proof.

Notice that since $\mathbb{Z}_{3} \times S U(5) \subset S U(9)$, we have $S U(5) \subset S U(9) / \mathbb{Z}_{3}$. Similarly $S U(9) / \mathbb{Z}_{3}$ contains $S U(6), S U(7)$, and $S U(8)$, and $\operatorname{Ad}(\varrho)$ induces complex conjugation on all of them. Now the $S U(6) \subset S U(9) / \mathbb{Z}_{3}$ is the same $S U(6)$ discussed previously. Since $\operatorname{Ad}(\varrho)$ is an automorphism of $S U(6)$, it is also an automorphism of $S U(2) \times S U(3)$. It is now clear that $S O(6)$ is precisely the subgroup of $S U(6)$ consisting of elements that centralise $\varrho$, and so we have

$$
C[S O(6)]=[S U(2) \times S U(3)] \rtimes \mathbb{Z}_{2} .
$$

This is, of course, very different to $C[S U(4)]=\operatorname{Spin}(10)$; the rank of $C[S O(6)]$ is only 3. This means, in particular, that string compactifications on manifolds of holonomy $S O(6)$ are of interest only if one can find another $U(1)$ factor in some other way. [See Gepner (1988).] 
Finally, $O(6)$ can be embedded in $S U(9)$ by

$$
s \rightarrow\left[\begin{array}{cc}
s & 0 \\
0 & \delta I_{3}
\end{array}\right]
$$

where $\delta=\operatorname{det} s$, for any $s \in O(6)$. This projects to an embedding of $O(6)$ in $S U(9) / \mathbb{Z}_{3}$. The centraliser is just the projection of the $S U(9)$ subgroup consisting of all matrices of the form $\left[\begin{array}{cc}\alpha I_{6} & 0 \\ 0 & u\end{array}\right]$, where $\alpha \in U(1), u \in U(3)$, and $\alpha^{6} \operatorname{det}(u)=1$. All such matrices can be expressed as $\left[\begin{array}{cc}\alpha I_{6} & 0 \\ 0 & \alpha^{-2} I_{3}\end{array}\right] \cdot\left[\begin{array}{cc}I_{6} & 0 \\ 0 & s\end{array}\right]$ for some $s \in S U(3)$ and so this group is isomorphic to $U(1) \times S U(3)$ (not $U(3)$ ). The $\mathbb{Z}_{3}$ in the centre of $S U(9)$ is contained in $U(1)$, and so the projection in $S U(9) / \mathbb{Z}_{3}$ is also isomorphic to $U(1)$ $\times S U(3)$. Since $O(6)$ consists of real matrices,

$$
C[O(6)]=[U(1) \times S U(3)] \rtimes \mathbb{Z}_{2} .
$$

$n=7$ : The possibilities for $\Psi(M)$ are $S O(7), O(7), G_{2}$, and $\mathbb{Z}_{2} \times G_{2}$. The first two may be embedded in $S U(9) / \mathbb{Z}_{3}$ and their centralisers computed in much the same way as $S O(6)$ and $O(6)$. The results are $C[S O(7)]=U(2) \rtimes \mathbb{Z}_{2}$ and $C[O(7)]=\mathbb{Z}_{2} \times O(2)$.

$G_{2}$ has a natural embedding in $E_{8}$ through $G_{2} \times F_{4}$. We cannot use Theorem (3.5) here directly, because $Z\left(G_{2}\right)=Z\left(G_{2} \times F_{4}\right)=Z E_{8}=\mathbb{Z}_{1}$. Instead we reason as follows. $G_{2}$ has maximal connected subgroups isomorphic to $S U(3)$ and $S O(4)$ respectively. These are also subgroups of the canonical $\operatorname{Spin}(6)$ in $E_{8}$; indeed, we have the following inclusions:

$$
\left\{\begin{array}{l}
S U(3) \\
S O(4)
\end{array}\right\}>G_{2} \longrightarrow \operatorname{Spin}(7) \rightarrow \operatorname{Spin}(16) / \mathbb{Z}_{2} \rightarrow E_{8}
$$

[See Salamon (1989); note that $\operatorname{Spin}(6)=S U(4)$ and that, since $G_{2}$ is the only connected Lie group with algebra $L G_{2}$, it follows that both $S O(7)$ and $\operatorname{Spin}(7)$ have subgroups isomorphic to $G_{2}$. ] Hence we see that the $S U(3)$ in $G_{2}$ is precisely the familiar canonical $S U(3)$ in $E_{8}$. Now the Dynkin diagram of $G_{2}$ has no symmetries. Proceeding just as in Theorem (8.1), we can show that there exists $\xi \in G_{2}$ such that $\operatorname{Ad}(\xi)$ induces complex conjugation on $S U(3)$, and therefore also on its $S U(2)$ subgroup. By Theorem (3.5), the centraliser of $S U(3)$ in $G_{2}$ is $\mathbb{Z}_{3}$, while that of $S U(2)$ is $S U(2)\left(\right.$ recall $\left.S O(4)=[S U(2) \times S U(2)] / \mathbb{Z}_{2}\right)$. Hence $\xi^{6}=\xi^{4}=1$, so $\xi^{2}=1$. Thus we have $S U(3) \rtimes \mathbb{Z}_{2} \subset G_{2}$. But this subgroup is not necessarily the same as the one discussed in Theorem (8.1) - we do not know whether $\xi=\zeta$. All we can say is that since $\operatorname{Ad}(\xi)$ induces an automorphism of $S U(3)$, it also induces an (involutive) outer automorphism of $E_{6}$. According to Theorem (8.3), this means that the $E_{8}$ centraliser of the $S U(3) \rtimes \mathbb{Z}_{2}$ subgroup of $G_{2}$ is isomorphic either to $S p(4) / \mathbb{Z}_{2}$ or to $F_{4}$. Hence $C G_{2}$ is contained in one of these. But since $G_{2}$ is embedded through $G_{2} \times F_{4}$, we have $F_{4} \subseteq C G_{2}$. As $S p(4) / \mathbb{Z}_{2}$ does not contain $F_{4}$, we have $C G_{2}=F_{4}$ precisely.

Finally, we embed the $\mathbb{Z}_{2}$ factor of $\mathbb{Z}_{2} \times G_{2}$ in $F_{4}$ as the centre of the $\operatorname{Spin}(9)$ subgroup of $F_{4}$. We leave it to the reader to prove that the $F_{4}$ centraliser of this $\mathbb{Z}_{2}$ is precisely $\operatorname{Spin}(9)$ itself. Hence $C\left(\mathbb{Z}_{2} \times G_{2}\right)=\operatorname{Spin}(9)$.

$n=8$ : The possibilities for $\Psi(M)$ are $S O(8), O(8), S U(4), \mathbb{Z}_{8} \cdot S U(4), S U(4) \rtimes \mathbb{Z}_{2}$, $\left[\mathbb{Z}_{8} \cdot S U(4)\right] \rtimes \mathbb{Z}_{2}, S p(2), \mathbb{Z}_{3} \times S p(2)$, and $\operatorname{Spin}(7)$. The first two can be embedded in $S U(9) / \mathbb{Z}_{3} ; S U(4)$ and $S U(4) \rtimes \mathbb{Z}_{2}$ have been treated at length; $S p(2)$ and $\mathbb{Z}_{3} \times S p(2)$ 
were discussed at the beginning of Sect. 8; and Spin(7) is covered by Theorem (7.1). Finally, $\mathbb{Z}_{8} \cdot S U(4)$ can be embedded in $E_{8}$ through

$$
\mathbb{Z}_{8} \cdot S U(4) \rightarrow U(1) \cdot S U(4)=U(4) \rightarrow S U(5) \rightarrow S U(5) \cdot S U(5),
$$

and $\left[\mathbb{Z}_{8} \cdot S U(4)\right] \rtimes \mathbb{Z}_{2}$ is, similarly, a subgroup of $S U(5) \rtimes \mathbb{Z}_{2}$. The centralisers can be computed by the usual methods, and they are listed in the Table below.

$n=9: S O(9)$ is a subgroup of $\left[S U(9) / \mathbb{Z}_{3}\right] \rtimes \mathbb{Z}_{2}$; it commutes with the $\mathbb{Z}_{3}$ centre of $S U(9) / \mathbb{Z}_{3}$ and also, because it is real, with $\mathbb{Z}_{2}$. Hence the centraliser is $\mathbb{Z}_{3} \rtimes \mathbb{Z}_{2}$, the dihedral group of order 6 . On the other hand, $O(9)$ is $\mathbb{Z}_{2} \times S O(9)$, and its centraliser is $\mathbb{Z}_{2}$.

$n=10$ : Here we begin to find holonomy groups, namely $S O(10)$ and $O(10)$, which are simply too large to be embedded in $E_{8}$. Hence we need only consider $S U(5)$ (with $C(S U(5))=S U(5))$ and $S U(5) \times \mathbb{Z}_{2}$ (with centraliser $S O(5)$ ).

$n=12$ : The holonomy groups that can be embedded in $E_{8}$ are $S U(6), S U(6) \rtimes \mathbb{Z}_{2}$, $\mathbb{Z}_{12} \cdot S U(6),\left[\mathbb{Z}_{12} \cdot S U(6)\right] \rtimes \mathbb{Z}_{2}, S p(3), \mathbb{Z}_{4} \cdot S p(3), \mathbb{Z}_{8} \cdot S p(3), Q_{8} \cdot S p(3), Q_{16} \cdot S p(3)$. These last five are the only ones that require further discussion. We know that $F_{4}$ has a maximal connected subgroup of the form $S U(2) \cdot S p(3)$, while $E_{7}$ has a maximal connected subgroup isomorphic to $S p(3) \times G_{2}$. In fact $S U(2) \cdot E_{7}$ and $G_{2} \times F_{4}$ are the only maximal connected subgroups of $E_{8}$ that contain $G_{2} \times S U(2) \cdot S p(3)$. Since

$$
G_{2} \times S U(2) \cdot S p(3) \subseteq S p(3) \cdot C[S p(3)],
$$

and since $F_{4}$ and $E_{7}$ certainly do not centralise their $S p(3)$ subgroup, we have $C_{0}[S p(3)]=S U(2) \times G_{2}$. All that remains is to deal with discrete factors, if any. Let $c$ be an element of $C[S p(3)]$ that is not an element of $C_{0}[S p(3)]$. Then $c$ is an element of a disconnected group with $G_{2} \times S U(2) \cdot S p(3)$ as its identity component. Since the identity component of a Lie group is always normal, $\operatorname{Ad}(c)$ either acts trivially on $G_{2} \times S U(2) \cdot S p(3)$ or induces an outer automorphism on it. Using the method of Lemma (8.2), one can show that, in the latter case, $\operatorname{Ad}(c)$ would restrict to an outer automorphism of $S p(3)$. But $S p(3)$ has no such automorphism. Therefore $c$ centralises $G_{2} \times S U(2) \cdot S p(3)$. In particular $c$ centralises $G_{2}$, so $c \in F_{4}$ and it centralises $S U(2) \cdot S p(3)$ in $F_{4}$. By Theorem (3.5), c lies in the centre of $S U(2) \cdot S p(3)$, so $c \in S U(2)$. But this contradicts the definition of $c$; hence $C[S p(3)]=C_{0}[S p(3)$ $=S U(2) \times G_{2}$. Embedding $\mathbb{Z}_{4}, \mathbb{Z}_{8}, Q_{8}$, and $Q_{16}$ in $S U(2)$, one can now easily compute the centralisers of the other four holonomy groups with $S p(3)$ as identity component.

$n=14: S U(7)$ and $S U(7) \rtimes \mathbb{Z}_{2}$ can be embedded in $\left[S U(9) / \mathbb{Z}_{3}\right] \rtimes \mathbb{Z}_{2}$.

$n=16: S U(8), \mathbb{Z}_{16} \cdot S U(8), S U(8) \rtimes \mathbb{Z}_{2}$, and $\left[\mathbb{Z}_{16} \cdot S U(8)\right] \rtimes \mathbb{Z}_{2}$ can all be embedded in $\left.\left[S U(9) / \mathbb{Z}_{3}\right] \times \mathbb{Z}_{2}\right], S p(4)$ can be embedded in $S U(8)$; it can be characterised as the subgroup of $S U(8)$ consisting of matrices $S$ such that $S^{T} J S=J$, where $J=\left[\begin{array}{cc}0 & -I_{4} \\ I_{4} & 0\end{array}\right]$. This can be written as $J S J^{-1}=\bar{S}$. Let $\varrho$ be defined as in Lemma (9.1). Then clearly $\operatorname{Ad}(\varrho)$ induces complex conjugation on $S U(8)$, and so $J S J^{-1}=\operatorname{Ad}(\varrho) S$ for all $S$ in $S p(4)$; in short, $S p(4)$ is just the fixed point set of $\operatorname{Ad}(\varrho J)$. Hence $\varrho J \in C[S p(4)]$, and one finds $C[S p(4)]=\mathbb{Z}_{4} \cdot U(1)$, where $\mathbb{Z}_{4}$ is generated by $\varrho J$, where $U(1)$ denotes the projection of $U(1)$ embedded in $S U(9)$ by 
$\alpha \rightarrow\left[\begin{array}{cc}\alpha I_{8} & 0 \\ 0 & \alpha^{-8}\end{array}\right]$, and where the dot indicates the product of subgroups in $E_{8} \cdot\left(\mathbb{Z}_{4}\right.$ does not centralise $U(1)$, but nor is the product semi-direct, because the intersection is non-trivial.) The final possibility, $\mathbb{Z}_{5} \times S p(4)$, is embedded by putting $\mathbb{Z}_{5}$ inside $U(1)$. Since $\varrho$ does not commute with $\mathbb{Z}_{5}$, the centraliser is simply $U(1)$.

To summarise, the following table lists every holonomy group sufficiently small to be embedded in $E_{8}$, together with the corresponding interior symmetry group (that is, the centraliser in $E_{8}$ ). For the convenience of the reader, the holonomy groups are arranged according to whether the corresponding manifold is hyperKähler, Kähler but not hyperKähler, orientable but not Kähler, or nonorientable.

\section{Conclusion}

The list of possible gauge subgroups of $E_{8}$ given in the table may seem rather lengthy. One should bear in mind, however, that we are allowing the subgroups to be disconnected, and that $E_{8}$ has infinitely many different isomorphism classes of disconnected subgroups. On the other hand, many of the groups that arise in this way are of limited interest as gauge groups. Indeed, perhaps the most striking outcome of Sect. 9 is the fact that the familiar $E_{6}$ gauge group which arises in string compactifications is actually one of the very few viable gauge subgroups of $E_{8}$. If we adhere to a conventional, "grand unified" approach, then the only real alternatives to manifolds with $S U(3)$ holonomy are those with holonomy $S O(4)$, $\mathbb{Z}_{4} \cdot S U(2), Q_{8} \cdot S U(2), S U(4)$, and $\mathbb{Z}_{8} \cdot S U(4)$. All other compact Ricci-flat locally irreducible manifolds lead to gauge groups which are either of rank $<4$, which only have self-conjugate representations, or which are known to have phenomenological difficulties. If we further require that the formula $\#=\frac{1}{2}|\chi+\tau|$ should lead to precisely three generations, then $\mathbb{Z}_{4} \cdot S U(2)$ and $Q_{8} \cdot S U(2)$ are ruled out, as follows. It can be shown that the universal covering manifold of any compact manifold with holonomy $\mathbb{Z}_{4} \cdot S U(2)$ or $Q_{8} \cdot S U(2)$ must itself be compact and possess a metric with holonomy $S U(2)$. Such a manifold must be diffeomorphic to a $K_{3}$ surface. For $K_{3}, \chi+\tau=8$, and so $K_{3}$ cannot cover a manifold which gives rise to precisely 3 generations.

If we go further and require that $M$ be multiply connected (so that we can use the Hosotani gauge symmetry breaking mechanism), then $S U(4)$ is also eliminated, because any compact manifold with holonomy precisely $S U(4)$ must be simply connected (Beauville, 1983). One should also note that a compact manifold with holonomy $\mathbb{Z}_{8} \cdot S U(4)$ must have $\mathbb{Z}_{2}$ as its fundamental group, so there is very little leeway in that case. Finally, no example of a compact Ricci-flat 4-manifold with holonomy $S O(4)$ is known. It is conceivable that none exists - though in view of the peculiarities of four-dimensional geometry, one cannot be confident of this.

We conclude, then, that within the framework of the usual assumptions, the familiar Calabi-Yau manifolds with $\Psi(M)=S U(3)$ are almost certainly the only compact Ricci-flat manifolds that need to be considered. Of course, one might be prepared to relax some of these conditions: for example, one could try to use Witten's topological approach to account for only two of the observed generations, seeking elsewhere for the origin of the third. Again, we saw that manifolds with holonomy $S O(6)$ give rise to a gauge group of the form $[S U(2) \times S U(3)] \rtimes \mathbb{Z}_{2}$; 
Table 1. Interior symmetry groups of Ricci-flat compact manifolds with holonomy groups extended to $E_{8}$

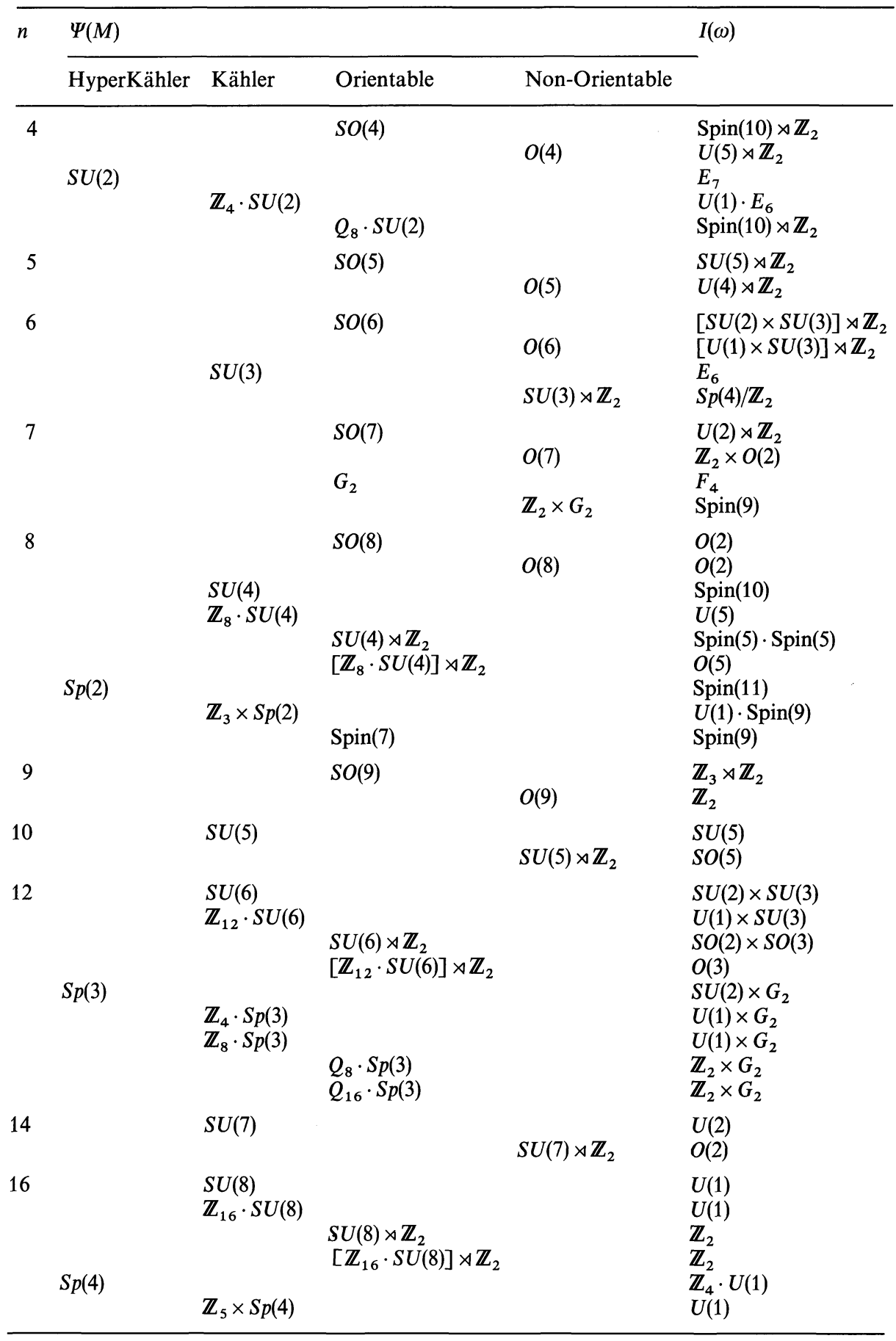


if a $U(1)$ factor could be found in some other way, this would give us the standard group directly, without any need for grand unification. This last possibility merits further attention, and will be considered elsewhere.

Acknowledgements. The author is grateful to Dr. Helmer Aslaksen and to Dr. Lang Mong Lung for helpful discussions on the root system of $E_{8}$.

\section{References}

Beauville, A.: Variétés Kähleriennes dont la première classe de Chern est nulle. J. Diff. Geom. 18, 755-782 (1983)

Berger, M.: Sur les groupes d'holonomie des variétés à connexion affine et des variétés Riemanniennes. Bull. Soc. Math. France 83, 279-330 (1955)

Besse, A.: Einstein Manifolds. Berlin, Heidelberg, New York: Springer 1987

Bröcker, T., tomDieck, T.: Representations of Compact Lie Groups. Berlin, Heidelberg, New York: Springer 1985

Candelas, P., Horowitz, G.T., Strominger, A., Witten, E.: Vacuum configurations for superstrings. Nucl. Phys. B 258, 46-74 (1985)

Chan, H.M., Tsou, S.T.: Monopole charges in unified gauge theories. Nucl. Phys. B 189, 364-380 (1981)

Curtis, M.L.: Matrix Groups. Berlin, Heidelberg, New York: Springer 1984

Ellis, J., Enqvist, K., Kalara, S., Nanopoulos, D.V., Olive, K.A.: Phenomenological studies of rank-5 Calabi-Yau manifolds. Nucl. Phys. B 306, $445-469$ (1988)

Fischer, A.E.: The internal symmetry group of a connection on a principal fiber bundle with applications to gauge field theories. Commun. Math. Phys. 113, 231-262 (1987)

Gepner, D.: Space-time supersymmetry in compactified string theory and superconformal models. Nucl. Phys. B 296, 757-778 (1988)

Green, M.B., Schwarz, J.H., Witten, E.: Superstring theory, Vol. II. London: Cambridge University Press 1987

Helgason, S.: Differential geometry, Lie groups, and symmetric spaces. New York, San Francisco, London: Academic Press 1978

Humphreys, J.E.: Introduction to Lie algebras and representation theory. Berlin, Heidelberg, New York: Springer 1972

Husemoller, D.: Fibre bundles. Berlin, Heidelberg, New York: Springer 1975

Kobayashi, S., Nomizu, K.: Foundations of differential geometry, Vol. I. New York, London, Sydney: Interscience 1963

McInnes, B.: Hosotani breaking of $E_{6}$ to a subgroup of rank five. J. Math. Phys. 31, 2094-2104 (1990)

McInnes, B.: Methods of holonomy theory for Ricci-flat Riemannian manifolds. CERN preprint, CERN-Th.5706/90

Salamon, S.: Riemannian geometry and holonomy groups. Harlow, New York: Longman/John Wiley 1989

Slansky, R.: Group theory for unified model building. Phys. Rep. 79, 1-128 (1981)

Vilenkin, A.: Cosmic strings and domain walls. Phys. Rep. 121, 263-315 (1985)

Witten, E.: Fermion quantum numbers in Kaluza-Klein Theory. In: Khuri, N. et al. (eds.) Shelter Island II Conference on Quantum Field Theory and the Fundamental Problems of Physics, Shelter Island, NY, 1983, pp. 227-277. Proceedings. Cambridge, Mass.: MIT Press 1985

Wolf, J.A.: Spaces of constant curvature. New York, St. Louis, San Francisco, Toronto, London, Sydney: McGraw-Hill 1967 